\title{
Tilted Fibre Bragg Grating sensors. Application in monitoring of the chemical fabrication of nanometer metal films
}

\author{
by \\ Aliaksandr Bialiayeu

\begin{abstract}
A Thesis submitted to
the Faculty of Graduate Studies and Research

in partial fulfilment of

the requirements for the degree of

Master of Applied Science

Ottawa-Carleton Institute for

Electrical and Computer Engineering
\end{abstract} \\ Department of Electronics \\ Carleton University \\ Ottawa, Ontario, Canada \\ March 2010
}




$\begin{array}{ll}\begin{array}{l}\text { Library and Archives } \\ \text { Canada }\end{array} & \begin{array}{l}\text { Bibliothèque et } \\ \text { Archives Canada }\end{array} \\ \begin{array}{l}\text { Published Heritage } \\ \text { Branch }\end{array} & \begin{array}{l}\text { Direction du } \\ \text { Patrimoine de l'édition }\end{array} \\ \begin{array}{l}\text { 395 Wellington Street } \\ \text { Ottawa ON K1A ON4 } \\ \text { Canada }\end{array} & \begin{array}{l}\text { 395, rue Wellington } \\ \text { Ottawa ON K1A ON4 } \\ \text { Canada }\end{array}\end{array}$

Your file Votre référence

ISBN: 978-0-494-71529-1

Our file Notre référence

ISBN: 978-0-494-71529-1

NOTICE:

AVIS:

The author has granted a nonexclusive license allowing Library and Archives Canada to reproduce, publish, archive, preserve, conserve, communicate to the public by telecommunication or on the Internet, loan, distribute and sell theses worldwide, for commercial or noncommercial purposes, in microform, paper, electronic and/or any other formats.

The author retains copyright ownership and moral rights in this thesis. Neither the thesis nor substantial extracts from it may be printed or otherwise reproduced without the author's permission.

L'auteur a accordé une licence non exclusive permettant à la Bibliothèque et Archives Canada de reproduire, publier, archiver, sauvegarder, conserver, transmettre au public par télécommunication ou par l'Internet, prêter, distribuer et vendre des thèses partout dans le monde, à des fins commerciales ou autres, sur support microforme, papier, électronique et/ou autres formats.

L'auteur conserve la propriété du droit d'auteur et des droits moraux qui protège cette thèse. Ni la thèse ni des extraits substantiels de celle-ci ne doivent être imprimés ou autrement reproduits sans son autorisation.
In compliance with the Canadian Privacy Act some supporting forms may have been removed from this thesis.

While these forms may be included in the document page count, their removal does not represent any loss of content from the thesis.
Conformément à la loi canadienne sur la protection de la vie privée, quelques formulaires secondaires ont été enlevés de cette thèse.

Bien que ces formulaires aient inclus dans la pagination, il n'y aura aucun contenu manquant. 
Copyright (C)

2010 - Aliaksandr Bialiayeu 


\section{Abstract}

An accurate and efficient sensing platform based on a tilted fibre Bragg gratings (TFBG) sensor, in particular concerning the monitoring of the chemical fabrication of nanometer metal films is presented.

TFBGs have grating planes that are tilted relative to the fiber's transverse axis thereby causing the excitation of several tens of cladding mode resonances, each uniquely addressed by its wavelength. The large differential sensitivity of the core and cladding modes to various perturbations allows the development of very accurate fiber sensors with a unique set of advantages and wide operating range.

In presented work the TFBG is used to monitor the fabrication of the nanoscale metal layers on the outside of the fibre cladding, that will eventually be used for sensing by metal-coated fibres. The sensor calibration is done by relating the fabricated metal film thickness, measured by Atomic Force Microscopy, to the spectral response of the sensor. Measurements using linearly polarized were determined to be crucial for the accurate monitoring of gold coatings with thicknesses ranging from 1 to $50 \mathrm{~nm}$.

The developed sensing platform is capable of acquiring the optical response of the sensor at 50 different states of linearly polarized light, within a 10 second interval. Changes in metal film thickness as small as $1 \mathrm{~nm}$ were detected during the real-time monitoring of an electroless metal plating process.

This possibility opens the way of creating TFBG-based surface plasmon resonance sensors, where the thickness of the metal coating is precisely tuned to obtain the 
plasmon resonance in the working range of the TFBG sensor.

In order to get a physical insight into the processes occurring in the sensor, a numerical method was developed for a fast and accurate solution to the sensor's electromagnetic field structure problem, and a mode coupling technique was introduced for the theoretical prediction of the sensor's spectral response. 


\section{Acknowledgments}

I would like to thank my supervisor Professor Jacques Albert for his support, patience, help and guidance throughout my Masters program at Carleton University.

I would also like to extend sincere thanks to Professor Anatoli Ianoul for his help and guidance, as well for spending a lot time helping me with the experimental part of the presented work.

I would like to thank Albane Laronche, Yanina Shevcheko, Tuan Guo and Graham Galway for helping me in the lab with equipment and experiment setup.

I am grateful to my colleagues Peter Krug and Alexander Andreyuk for many fruitful discussions.

Finally, I acknowledge the generous financial support of Carleton University and my supervisor Professor Jacques Albert. 


\section{Preface}

This thesis is organized as follows:

Chapter 1 presents background material relating to the fibre grating technology, grating writing process and mechanism of fibre photosensitivity.

Chapter 2 introduces the theoretical background necessary to understand the Bragg grating sensor operation principles along with a short introduction to the basic properties of Bragg gratings. The mathematical modelling of the grating is based on the coupled-mode theory.

In Chapter 3 the theory behind modes in multilayer cylindrical structures is reviewed. The vectorial mode transfer matrix method is derived.

Chapter 4 presents a developed numerical method for a fast and accurate solution to the fibre modes problem in multilayer step-index structures.

Experimental results are presented in Chapter 5.

Finally, conclusions from this research and suggestions for further research are presented in Chapter 6. 


\section{Table of Contents}

Abstract $\quad$ iv

Acknowledgments vi vi

Preface vii

Table of Contents viii

List of Figures $\quad$ xi

Nomenclature $\quad$ XV

1 Introduction to fibre Bragg gratings 1

1.1 Fabrication methods ................... 1

1.2 Mechanism of Photosensitivity . . . . . . . . . . . 6

1.2.1 Introduction to Photosensitivity . . . . . . . . . . . . 6

1.2.2 Models of Photosensitivity . . . . . . . . . . . . . 7

1.2 .3 Defects . . . . . . . . . . . . . . . 8

1.3 Fibre Bragg Grating Operation Principles . . . . . . . . . . . . . . . 10

2 Mode Couplings in Optical Fibres with a Bragg Grating 12

2.1 Introduction . . . . . . . . . . . . . . . . . . 12

2.2 General Derivation of Multi-Mode Coupling . . . . . . . . . . 14 
2.3 Bragg Gratings Spectrum Calculation . . . . . . . . . . . . 17

2.4 Bragg Grating Types . . . . . . . . . . . . . . . . . . 21

2.5 Mode Coupling in a Tilted Fibre Bragg Grating . . . . . . . . . . . 23

3 Optical Modes in a Multilayer Step-Index Cylindrical Waveguide 26

3.1 Electromagnetic Fields in

Cylindrical Waveguides . . . . . . . . . . . . . . 26

3.2 Boundary conditions . . . . . . . . . . . . . . . 33

3.3 Matrix method . . . . . . . . . . . . . . 37

4 Numerical Simulation $\quad 41$

4.1 Introduction . . . . . . . . . . . . . . 41

4.2 Numerical integration method . . . . . . . . . . . . . . 43

4.3 Eigenvalues search algorithm . . . . . . . . . . . . . 45

4.4 Results and Discussion . . . . . . . . . . . . . . . . . . 46

5 TFBG sensor for nanoscale metal films $\quad 48$

5.1 Introduction . . . . . . . . . . . . . . . . . . . . 48

5.2 Experiment Outline . . . . . . . . . . . . . . . . . 49

5.3 Metal film deposition . . . . . . . . . . . . . . . . 50

5.4 Optical system and TFBG response . . . . . . . . . . . . 53

5.5 Metal film thickness estimation . . . . . . . . . . . . 59

5.5 .1 Principles of AFM operation . . . . . . . . . . 59

5.5.2 AFM Measurement technique ............. 64

5.6 Results and Discussion . . . . . . . . . . . . . . . . 70

6 Conclusions and Future Work $\quad 72$

$\begin{array}{ll}\text { List of References } & 75\end{array}$ 
Appendix A Listing of program for analysing multilayer cylindrical structures 


\section{List of Figures}

1 Bragg grating written with standing wave . . . . . . . . . 1

2 Schematic of interferometric configuration used by Meltz in $1989 \ldots 2$

3 Interference pattern causing Bragg grating formation . . . . . . . . 3

$4 \quad$ FBG written with phase mask . . . . . . . . . . . . . 5

5 A fibre Brag grating operation principles . . . . . . . . . . . . . 10

6 Bragg grating momentum diagram of contra-directional coupling for the general case of phase mismatch ... . . . . . . . . 18

7 Long period Bragg grating momentum diagram of co-directional coupling 18

8 The reflectivity of a uniform grating as a function of phase mismatch. 20

$9 \quad$ Fibre gratings types:

(a) uniform with positive-only index change, (b) Gaussian-apodized,

(c) raised-cosine-apodized with zero-dc index change, (d) chirped,

(e) discrete phase shift, (f) superstructure. . . . . . . . . . . . 21

10 TFBG momentum vector diagram of contra-directional coupling. . . . 24

11 Typical TFBG spectrum $\left(\theta=6^{0}\right) \ldots \ldots \ldots . \ldots \ldots$

12 Schematic structure of 4 layer circular waveguide . . . . . . . . . 27

13 Schematic representation of the 4th order Runge-Kutta method . . . 44

14 The graphical user interface of the program. . . . . . . . . . . . . 47

15 Schematic representation of AuNPs assisted electroless plating. . . . . 50

16 AFM scan of the fibre coated with AuNPs and with the gold film . . 52 
17 Image of the fibre coated with the gold film taken from an optical microscope. ....................... 53

18 Schematic diagram of the optical system. . . . . . . . . . . 53

19 (a) Typical transmission spectrum of a weakly tilted FBG, (b) a 3D representation of a set of the TFBG spectra taken at different deposi-

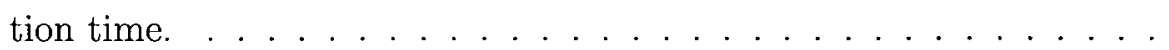

20 Evolution of the spectrum envelope during the whole process of deposition. ............................ 55

21 Density and 3D plot of individual resonances. . . . . . . . . . 55

22 Schematic diagram of the optical system with a polarizer controller added. . . . . . . . . . . . . . . . 56

23 The density plot representation of spectra acquired for the first $30 \mathrm{~min}$ of the gold film deposition . . . . . . . . . . . . . . . . 57

24 Density plot of the spectrum evolution for the P3 polarization state $(\lambda=1530 . .1560 \mathrm{~nm}, t=1 . .1800 \mathrm{sec}.) \ldots \ldots \ldots$

25 Evolution of individual resonance near $1531 \mathrm{~nm}$ for P1,P2,P3 polarization states . . . . . . . . . . . . . . . . . . . 59

26 The tip is attached to a cantilever, and is $\mathrm{x}, \mathrm{y}$-scanned over a surface. The cantilever deflection due to tip-surface interactions is monitored by a photodiode. . . . . . . . . . . . . . . . 60

27 The scratched metallic film and its height estimation . . . . . . 65

28 Flattened AFM Image . . . . . . . . . . . . . . . . . 66

29 Histogram representation of a film surface. . . . . . . . . . . . . . . 67

30 Histogram of AFM scans of the gold film with different exposure time. 68

31 Histograms filtered with a low pass "brick" filter. . . . . . . . . 69 
32 Zoom of Figure 31 (Intersections with the "height" axis gives the value of the gold film thickness measured from the film surface to the fibre substrate). . . . . . . . . . . . . . . 69

33 The gold film thickness vs deposition time, with linear and cubic interpolation. ........................ 70

34 Gold film thickness vs intensity for one particular cladding mode resonance at $1531 \mathrm{~nm}$. The line represents the second order polynomial interpolation. ...................... 71

35 Possible SPR detection at P2 polarization state (black line) after 40 min of gold film deposition . . . . . . . . . . . . . 


\section{Nomenclature}

$\vec{E}, \vec{H} \quad$ Electric and Magnetic fields

$\epsilon_{0} \quad$ Vacuum permittivity

$\epsilon \quad$ Material permittivity

$\mu_{0} \quad$ Vacuum permeability

$\mu \quad$ Material permeability

c Velocity of light in vacuum

$\omega \quad$ Angular Frequency

$\beta \quad$ Propagation constant

$n \quad$ Refractive index

$n_{c} \quad$ Core refractive index in a fibre

$n_{e f f} \quad$ Fibre effective refractive index

$k_{0} \quad$ Free space wave propagating constant

$\overrightarrow{k_{i}} \quad$ Incident wave vectors

$\overrightarrow{k_{s}} \quad$ Scattered wave vectors

$\overrightarrow{k_{B}} \quad$ Grating wave vectors 
$\nabla * \quad$ The Divergence operator

$\nabla \times \quad$ The Curl operator

$\partial_{t} \quad$ Partial derivative with respect to variable $t$

$\Delta \epsilon \quad$ Dielectric perturbation

$\Delta \beta \quad$ Phase mismatch

$\delta_{m n} \quad$ Dirac delta

$d_{m n} \quad$ Matrix coefficients of the coupling strength

$m \quad$ Mode angular symmetry number

$M^{(i)} \quad$ Transfer matrix for $i$ th layer

$\lambda \quad$ Wavelength

$\lambda_{B} \quad$ Bragg grating wavelength

$\Lambda \quad$ Grating period

$L \quad$ Length of the fibre

$N \quad$ Number of the grating periods

I Intensity

$R \quad$ Reflectivity

T Transmissivity

$J_{m}, Y_{m}$ Bessel functions of the first and the second kind

$I_{m}, K_{m}$ Modified Bessel functions of the first and the second kind 


\title{
Acronyms
}

\author{
AFM Atomic Force Microscope \\ APTMS ((3-aminopropyl)trimethoxysilane \\ AuNPs Gold Nanoparticles \\ EM Electromagnetic field \\ FBG Fibre Bragg Grating \\ I R Infrared Radiation \\ LPBG Long-Period Bragg Grating \\ ODE Ordinary differential Equation \\ SPR Surface Plasmon Resonance \\ TFBG Tilted Fibre Bragg Grating \\ UV UltraViolet (radiation)
}




\section{Chapter 1}

\section{Introduction to fibre Bragg gratings}

\section{$1.1 \quad$ Fabrication methods}

The formation of permanent gratings by photosensitivity in an optical fibre was discovered by Ken Hill 1978 [1] at the Canadian Communication Research Center. They excited a germania-doped optical fibre with intense argon-ion laser radiation at 488 $\mathrm{nm}$ and observed formation of refractive-index gratings due to the exposure of the core to the interference pattern of oppositely propagating modes (Figure 1).

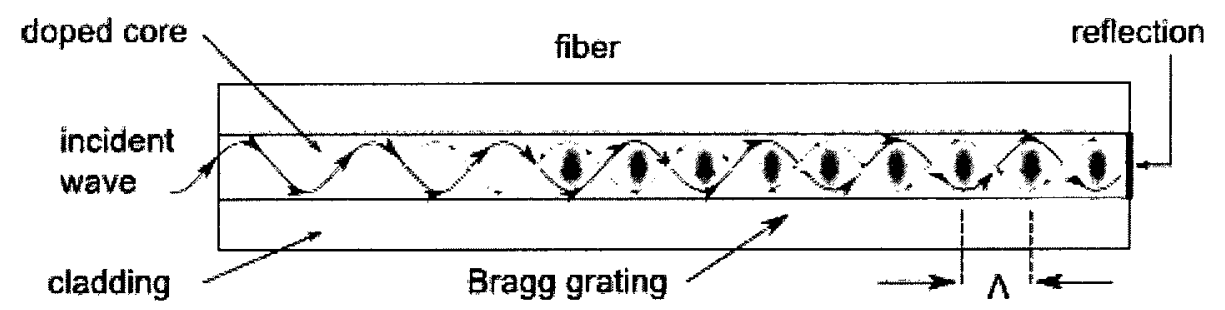

Figure 1: Bragg grating written with standing wave

Photosensitivity of germania-doped silica causes the index of refraction to be increased to a much greater extent at position where constructive interference results in a maximum of laser intensity. Investigations by Lam and Garside [2] showed that the grating strength increased as the square of the writing power, which suggested a two-photon process. 
It was suggested that the core refractive index modulation is the result of holographic process where coherent modes are coupled and launched into the fibre core causing a two-photon absorption process. Based on this observation Meltz [3] recognized that process of grating formation could be made more efficient if it were a one-photon process corresponding to the germania oxygen vacancy defect band.

In the experiment of Meltz the fibre was illuminated from the side with two intersecting coherent ultraviolet laser beams of wavelength $244 \mathrm{~nm}$, (see Figure 2 [3]), which corresponds to germania oxygen-vacancy defect band. This method worked since fibre cladding is transparent to UV light, whereas fibre core is highly absorbing of this radiation.

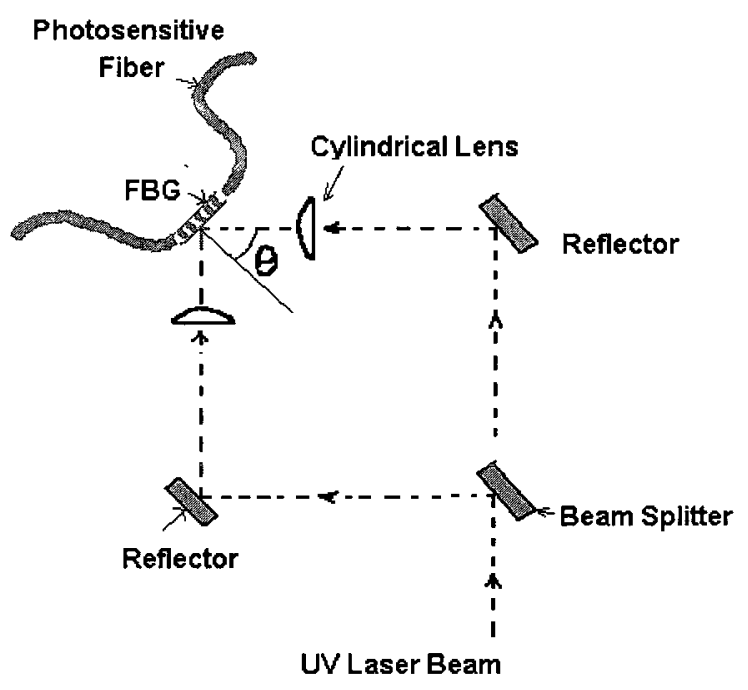

Figure 2: Schematic of interferometric configuration used by Meltz in 1989

The interferometer of Figure 2 consists of an optical beam splitter and two mirrors which recombine both beams yielding interference. This technique is most sensitive to alignment as variation between two beams paths must lay within the coherence length of the laser, which is approximately $200 \mu m$ for eximer lasers.

The Bragg wavelength $\lambda_{B}$ is the wavelength of the reflection maximum in the 
fibre and is related to the grating period and the effective refractive index $n_{\text {eff }}$ by the Bragg condition:

$$
\lambda_{B}=2 n_{e f f} \Lambda
$$

Spatial period of photo-induced perturbation can be selected by changing the angle between the interfering beams (Figure 2). This permits fabrication of custom Bragg gratings operating at much longer wavelengths than the writing wavelength (Figure 3).

$$
\Lambda=\frac{\lambda}{2 n_{c} \sin (\theta)}
$$

Where

$\Lambda$ is the Bragg grating period,

$n_{c}$ is the refractive index of the fibre's core,

$\theta$ is the angles of incidence of the UV writing beam,

$\lambda$ is the wavelength of the writing radiation.

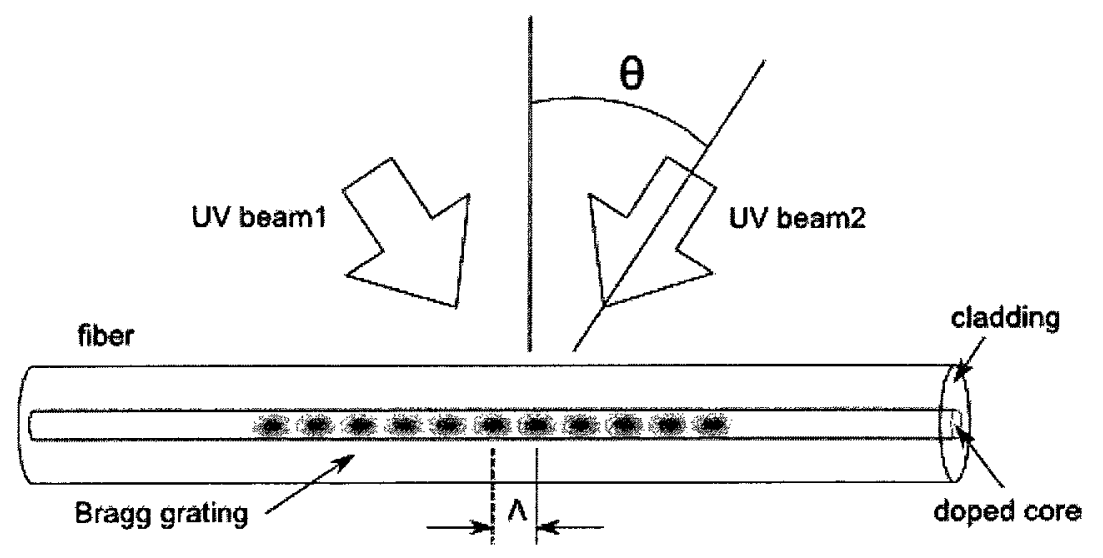

Figure 3: Interference pattern causing Bragg grating formation

The new FBG writing technique based on phase mask was introduced in 1993 [4] 
(see Figure 4). The idea was to use a phase mask transparent to UV laser to spatially modulate the phase of a UV beam and get interference pattern from diffracted UV field. Phase masks are a transparent to UV laser thin slab of silica glass into which is etched periodic structure.

There are two possibilities to implement phase mask in order to obtain $+1 /-1$ or $0 /-1$ diffraction beams. In the $+1 /-1$ configuration, the UV radiation is directed with normal incidence at the phase mask, and the period of the FBG is exactly one half of the period of the phase mask grating. In the $0 /-1$ configuration, the UV radiation is directed at the phase mask with a specially chosen angle of incidence, and the period of the FBG is equal to the period of the phase mask grating.

The shape of the periodic pattern of the phase mask might be chosen to approximate a square wave profile, in order to obtain diffraction pattern with only +1 and -1 diffraction orders and nulled zero-order diffracted beam. Also, the intensity in any higher orders $(= \pm 2, \pm 3$, etc.), if such orders are present, is minimized [4].

The phase mask technique allows to tune the writing wavelength to the peak of material photosensitive, as the grating period is independent on the writing wavelength.

The phase mask technique replaces the most of the optics used in the interferometric method with single mask thus simplifies the manufacture of FBGs through easier alignment and also lower coherence demands on the laser beam.

Krypton-fluoride and Argon fluoride excimer lasers are usually used to produce FBG writing. The exposure required is typically a few minutes with laser intensities of 100 to $1000 \mathrm{~mJ} / \mathrm{cm}^{2}$ and pulse rates of 50 to $75 \mathrm{~Hz}$. Under these conditions it is possible to achieve the core refraction index variation in the range of $10^{-5}$ to $10^{-3}$ in germanium doped single mode optical fibre, dependent on the UV-exposure and the dopants in the fibre. To enhance the optical fibre's photosensitivity techniques such as hydrogen loading [5] can be used. Hydrogen diffusion makes the core more 


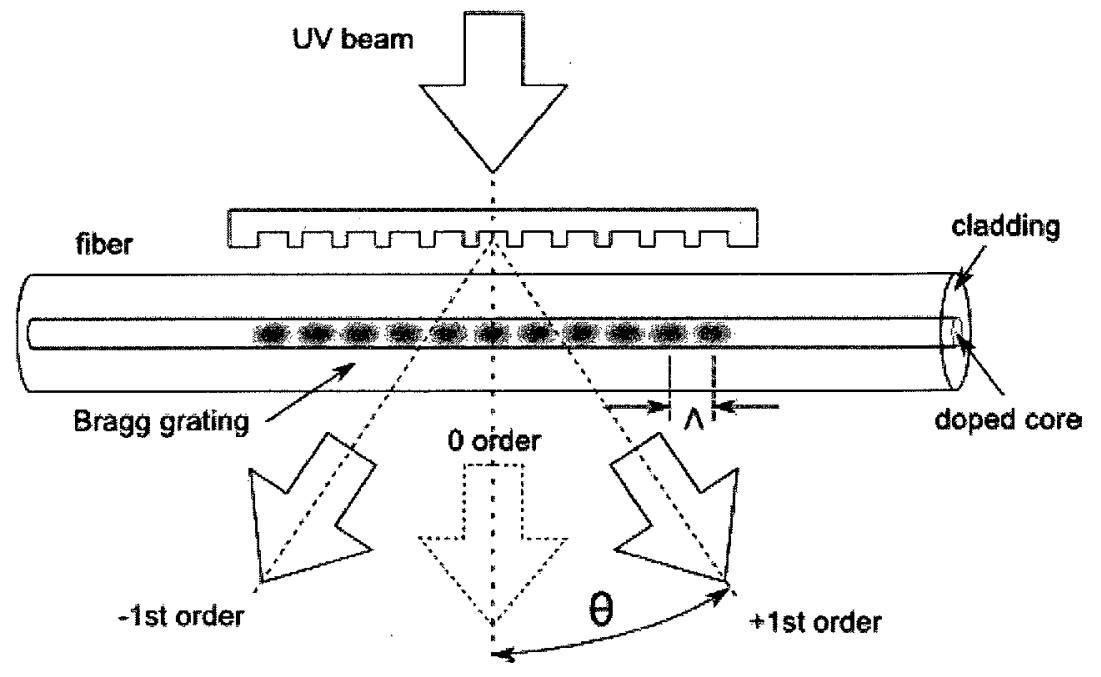

Figure 4: FBG written with phase mask

susceptible to UV laser radiation. That allows to achieve changes in refractive index of the order of $10^{-2}$. 


\subsection{Mechanism of Photosensitivity}

\subsubsection{Introduction to Photosensitivity}

Photosensitivity describes the phenomenon of refractive index perturbation produced by exposure of a material to optical radiation. The effect of interest is a stable, photoinduced change in refractive index in germanosilicate glasses, the primary material system used in telecommunications fibres.

While the precise mechanism causing the photo sensitive effect varies with material composition, processing and writing conditions, it is agreed that the phenomenon of UV-photosensitive response in germanosilicate glasses is linked to the presence of oxygen-deficient point defect centers associated with germanium in the glass structure. When a dopant like Ge is introduced during a metal organic chemical vapor deposition (MOCVD) process, the Ge atoms form bonds with both silicon and oxygen atoms. The Ge-Si bonds strongly absorb UV radiation between 240-248nm.

Photosensitivity requires coupling of the optical field to the material through allowed electronic states. The band gap of germanosilicate glasses in $6.9 \mathrm{eV}$, photosensitivity is observed at sub-band gap energy of $5 \mathrm{eV}[6]$ and relies on the existence of structural point defects. Photoinduced redistribution of carriers within these states is an initial step in the material photosensitive response. Depending upon the excitation conditions and relaxation pathways available, the photoexcitation of the material can result in a permanent redistribution of carriers within the density of states.

Due to the chemical reactions during glass forming at elevated temperature, defects are induced within the glass matrix [6]. Further more during fibre drawing,

atomic bonds in the glass can break and the glass becomes more inhomogeneous with randomly distributed broken bonds and trapped defects [6]. 


\subsubsection{Models of Photosensitivity}

There are two primary models of photo-induced refractive index changes in germanosilicate glasses: the color center model and the structural change model.

The color center model, first proposed by Hand and Russell [7], suggests that during irradiation the absorbed UV energy breaks the Ge-Si bond. The electrons released in this process are trapped at other defect sites, forming $\mathrm{Ge}(1)$ and $\mathrm{Ge}(2)$ color centers [8]. The added electron causes strong optical absorption at they color centers and change the refractive index at longer wavelengths. It was shown $[7,9]$ that using experimental data of fibre's absorption spectrum in the ultraviolet it is possible to successfully explain refractive index changes by applying the KramersKronig principle, i.e. relating the imaginary part of refractive index (absorption) in UV range with the real part of refractive index in visible or IR range.

The Structural Change model involves more dramatic optical modification of the glass structure. Local heating and higher order absorption processes can result in modification of the inherent stress state of the material and even its density compaction or volume expansion [10].

Compaction and relaxation of the glass network causes microscopic changes that shifts the band gap and UV absorption, thereby changing the macroscopic index of refraction.

The formation of permanent electric dipoles in the glass is also possible. The breakage of the Ge-Si bonds yields positively charged $\mathrm{Si}+$ sites (the Ge-E' centers) and a free electron. The $\mathrm{Si}+$ ion is fixed in the glass matrix, whereas the electron has enough energy to escape and get trapped at neighboring $\mathrm{Ge}(\mathrm{l})$ or $\mathrm{Ge}(2)$ sites. Each resulting dipole will produce a static dc polarization field that extends many molecular spacings out [7].

The overall effect is thought to be a combination of both models. 


\subsubsection{Defects}

Knowledge of the defect states in the glass is, thus, necessary to understand photosensitivity.

The disordered network glass structure present in germanosilicates can sustain a wide variety of structural defects, including vacancies, dangling bonds and dopant/impurity atoms [10].

Defects in the germanosilicates can be divided into two major groups: Germanium Oxygen Deficient Centers (GODC): Neutral Oxygen Monovacancy (NOMV), and Neutral Oxygen Divacancy (NODV) or Germanium Lone Pair Center (GLPC) (with absorption energy at $5.08 \mathrm{eV}$ and $5.16 \mathrm{eV}$ respectively [11]. In this case several valence germanium electrons are shared with oxygen and and rest is shared with another atom of germanium or silica [6].

Germanium Electron Centers (GEC): Ge(1), Ge(2), Ge E' with absorption energy at $4.6 \mathrm{eV}, 5.8 \mathrm{eV}$ and $6.4 \mathrm{eV}$ respectively [6]. Germanium does not share spare electrons and keeps them unbounded.

Disagreement within the literature still exists, however it is agreed [12] that at low intensities ( $<40 \mathrm{~mJ} / \mathrm{cm}^{2} /$ pulse) UV excitation $(5.0 \mathrm{eV})$ into the lower energy band is thought to be the initial step in the photo sensitive effect. Such an optical exposure results in the bleaching of the NOMV band and in the formation of Ge E' centers. [11]

At higher UV intensities, an alternative mechanism involving two-photon absorption from valence band states, linked to lone-pair electrons on bridging oxygens, to the conduction band is believed to result in the formation of germanium electron centers $\mathrm{Ge}(1)$ and $\mathrm{Ge}(2)$ centers and a self trapped hole center [11].

Under further illumination there is evidence that the GECs are converted to Ge E' centers and non-bridging oxygens $[11,12]$. 
It appears likely that all of the mechanisms proposed could contribute to the refractive index changes observed. The relative contributions, however, will depend on both the materials and the exposure conditions used.

Several approaches have been used to enhance the photosensitive response of these glasses through either direct material modification or optical exposure conditions, with varied success.

The nature of fabrication of glass is ideally suited to promoting defects. During fabrication, the fibre composition depends on the temperatures, pressures and atmospheric composition. If the fibre is immersed in pressurized hydrogen environment, UV exposure leads to dissociation of the $H_{2}$ molecules and reaction of $H+$ ions with Ge-based defects. Hydrogen loading may result in an increase of induced refractive index modulation from $3 * 10^{-5}$ to $10^{-2}$ [13]

To provide additional optically active sub-band-gap states different dopants and ion implantation may also be used [10]. 


\subsection{Fibre Bragg Grating Operation Principles}

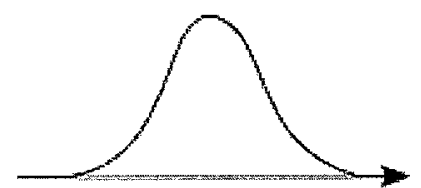

Input spectrum

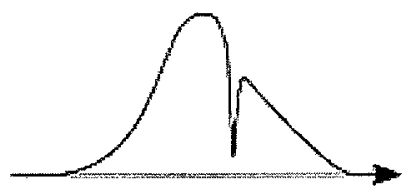

Transmitted spectrum
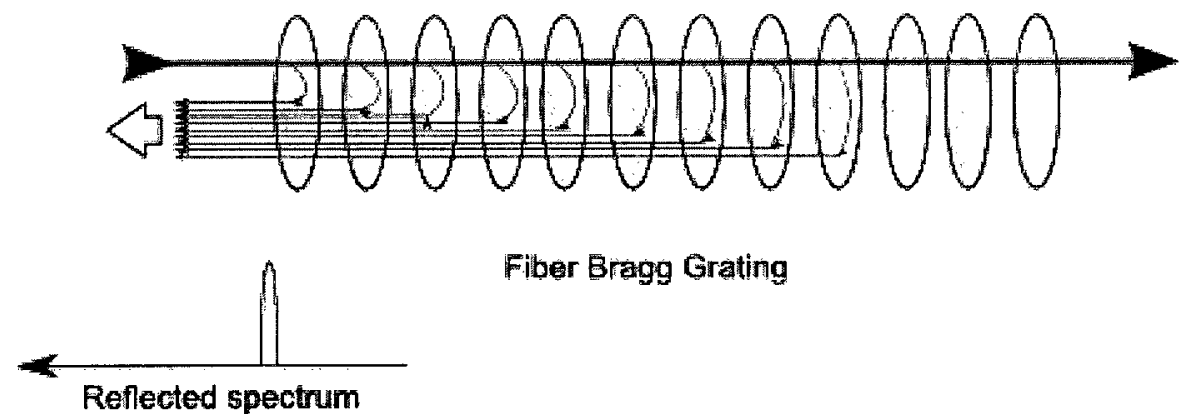

Fiber Bragg Grating

Figure 5: A fibre Brag grating operation principles

The principle of a fibre Bragg grating operation is similar to a multilayer dielectric optical mirror. Unlike having small number of layers with high refractive index variation, FBG has thousands of layers with small refractive index perturbation.

A FBG consist of a periodic modulation of refractive index in the core of a single mode fibre. The fundamental principle behind the operation of a FBG is Fresnel reflection. The light propagating along a single mode fibre encounters a refractive index perturbation and a small amount of it is scattered back at each interface layer. If each of these reflections are in phase (the phase matching condition), they will add coherently and result in a strong reflection up to more than 99 percent from the grating (Figure 5).

The phase matching condition occurs only if the Bragg condition of energy and momentum conservation are satisfied:

$$
\omega_{i}=\omega_{s}
$$




$$
\overrightarrow{k_{s}}+\overrightarrow{k_{B}}=\overrightarrow{k_{r}}
$$

Where

$\omega_{i}, \omega_{s}$ are the incident and scattered light frequencies.

$\overrightarrow{k_{i}}, \overrightarrow{k_{s}}$ are the incident and scattered wave vectors.

$\overrightarrow{k_{B}}=\frac{2 \pi}{\Lambda}$ is the Bragg wave vector, defined by grating period $\Lambda$.

Energy conditions $\omega_{i}=\omega_{s}$ is satisfied automatically as we have single mode in the core, while momentum conservation occurs only at a specific wavelength called the Bragg resonance wavelength $\Lambda_{B}$.

$$
\overrightarrow{k_{B}}=\frac{2 \pi}{\Lambda}=\frac{2 \pi}{\lambda_{B}} n_{e f f}
$$

The last equation yields

$$
\lambda_{B}=2 n_{e f f} \Lambda
$$

Where $\lambda_{B}$ is the Bragg resonance wavelength at which the light is reflected back by the grating, $n_{\text {eff }}$ is the effective refractive index of wave propagation.

Thus the grating effectively acts as a stop-band filter, reflecting light with wavelengths close to the Bragg wavelength, and transmitting the rest.

We will see in section 2.5 how these conditions are changed in the case of gratings with fringes that are tilted relative to the plane that is orthogonal to the fibre axis. 


\section{Chapter 2}

\section{Mode Couplings in Optical Fibres with a Bragg Grating}

\section{$2.1 \quad$ Introduction}

Coupled-mode theory has been applied extensively in guided-wave optics for the analysis of electromagnetic wave propagation and interaction with media. The coupledmode theory has also been useful in understanding nonlinear optical effects, such as second harmonic generation, parametric amplification, and modulation instability.

While other techniques are available for analyzing fibre Bragg grating spectral response, here we consider only the coupled-mode theory since it is straightforward and provides accurate models.

The coupled-mode theory was first developed by Pierce [14]. In the early 1970's the coupled-mode theory was introduced to guided-wave optics by Snyder and Yariv $[15,16]$ to analyze the mode coupling in optical waveguides.

It is known that any field in a waveguide might be represented as a linear superposition of its eigenmodes, which are usually found easily for an unperturbed structure [16]. Coupled-mode theory is based on the idea that energy can be transferred from one such propagating mode to another if small perturbation is introduced. The 
cross power terms appear as a result of modal nonorthogonality in slightly perturbed structure. Once these waveguide eigenmodes modes are determined, the amplitudes of the modes in the coupled-waveguide systems are governed by the coupled-mode equations.

A fibre Bragg grating contains periodic variations in the refractive index which acts as small perturbation and designed to achieve mode coupling. Mode coupling is defined by grating type and acts as intrinsic optical filter or coupler between core and cladding modes. The modal interaction can occur between modes within the same core, between core modes and cladding modes.

In this work we are interested in a core-cladding mode coupling. For this purpose we have to find all fibre transverse cross section eigenmodes profiles and calculate the grating-induced coupling coefficients between all these modes. As a result the coupled-mode analysis provides us a description of electromagnetic wave propagation and interaction in our sensor. 


\subsection{General Derivation of Multi-Mode Coupling}

Derivations of coupled-mode theory are usually done with the assumption of only two strongly coupled modes [17]. For our purpose we need to find coupling coefficients between all cladding-core modes. In this chapter we derive a general case of coupledmode theory.

For the unperturbed dielectric medium, homogeneous in the $z$ direction, the field $\vec{E}(x, y, z)$ might be represented as a linear combination of orthogonal modes $\overrightarrow{E_{m}}(x, y)$ with propagation constant $\beta_{m}$

$$
\vec{E}(x, y, z)=\sum_{m}^{N} c_{m} \overrightarrow{E_{m}}(x, y) e^{i\left(\omega t-\beta_{m} z\right)}
$$

Where $c_{m}$ is the amplitude of $m$-th mode, $N$ is the number of supported modes in the structure of interest.

Substituting it into Helmholtz equation

$$
\left(\nabla^{2}+\omega^{2} \mu \epsilon(x, y)\right) \vec{E}(x, y, z)=0
$$

where $\nabla^{2}=\nabla_{T}^{2}+\partial_{z}^{2}$, and considering that modes are linearly independent we may conclude that each of single modes also satisfies Helmholtz equation. Indeed otherwise all $c_{m}$ should be equal to zero.

$$
\left(\nabla_{T}^{2}+\omega^{2} \mu \epsilon(x, y)-\beta_{m}^{2}\right) \overrightarrow{E_{m}}(x, y)=0
$$

Now we assume that we have a small perturbation along $z$ axis defined as

$$
\epsilon(x, y, z)=\epsilon(x, y)+\Delta \epsilon(x, y, z)
$$

Applying perturbation theory we can represent our field as a linear combination of 
eigenmodes, but coupled in a way that energy might be transmitted from one mode to the other along propagation axis $z$.

$$
\vec{E}(x, y, z)=\sum_{m}^{N} c_{m}(z) \overrightarrow{E_{m}}(x, y) e^{i\left(\omega t-\beta_{m} z\right)}
$$

Substituting later equation in (9) we get

$$
\sum_{m}^{N}\left(c_{m} \nabla_{T}+\frac{d^{2} c_{m}}{d z^{2}}-i 2 \beta_{m} \frac{d c_{m}}{d z}-\beta_{m}^{2} c_{m}+c_{m} \omega^{2} \mu \epsilon\right) \overrightarrow{E_{m}} e^{i(\omega t-\beta z)}=0
$$

For a "weak" coupling resulting in small dielectric perturbation we have a "slow" variation of the mode amplitudes along the axis of propagation $z$

$$
\frac{d^{2} c_{m}}{d z^{2}} \ll \beta_{m} \frac{d c_{m}}{d z}
$$

Removing second derivative of $c(z)$ and combining $c$ term we get

$$
i 2 \sum_{m}^{N} \frac{d c_{m}}{d z} \beta_{m} \overrightarrow{E_{m}} e^{i(\omega t-\beta z)}=\sum_{m}^{N}\left(\nabla_{T}-\beta_{m}^{2}+\omega^{2} \mu \epsilon\right) c_{m} \overrightarrow{E_{m}} e^{i(\omega t-\beta z)} .
$$

Replacing $\epsilon$ with right part of (10) and using (9) we are left with equation

$$
i 2 \sum_{m}^{N} \beta_{m} \overrightarrow{E_{m}} e^{i\left(\omega t-\beta_{m} z\right)} \frac{d c_{m}}{d z}=\omega^{2} \mu \sum_{m}^{N} c_{m} \Delta \epsilon \overrightarrow{E_{m}} e^{i\left(\omega t-\beta_{m} z\right)} .
$$

Now let us take scalar product with complex conjugate field and integrate it over an infinite cross section.

$$
\begin{gathered}
i 2 \sum_{m}^{N} \beta_{m} e^{i\left(\beta_{n}-\beta_{m}\right) z} \int \overrightarrow{E_{m}} \overrightarrow{E_{n}^{*}} d x d y \frac{d c_{m}(z)}{d z}= \\
\omega^{2} \mu \sum_{m}^{N} c_{m}(z) e^{i\left(\beta_{n}-\beta_{m}\right) z} \int \overrightarrow{E_{m}} \Delta \epsilon(x, y, z) \overrightarrow{E_{n}^{*}} d x d y .
\end{gathered}
$$


As modes $\overrightarrow{E_{m}}$ are orthogonal

$$
\int \overrightarrow{E_{m}} \overrightarrow{E_{n}^{*}} d x d y \sim \delta_{m n}
$$

We can rewrite our equation

$$
i 2 \beta_{n} \frac{d c_{n}(z)}{d z}=\omega^{2} \mu \sum_{m}^{N} c_{m}(z) e^{i\left(\beta_{n}-\beta_{m}\right) z} \frac{\int \Delta \epsilon(x, y, z) \overrightarrow{E_{m}} \overrightarrow{E_{n}^{*}} d x d y}{\int \overrightarrow{E_{n}} \overrightarrow{E_{n}^{*}} d x d y}
$$

Finally

$$
\frac{d c_{n}(z)}{d z}=-\frac{i}{2} \frac{\omega^{2} \mu}{\beta_{n}} \sum_{m}^{N} d_{m n}(z) c_{m}(z) e^{-i\left(\beta_{m}-\beta_{n}\right) z}
$$

where

$$
d_{m n}(z)=\frac{\int \Delta \epsilon(x, y, z) \overrightarrow{E_{m}} \overrightarrow{E_{n}^{*}} d x d y}{\int \overrightarrow{E_{n}} \overrightarrow{E_{n}^{*}} d x d y}
$$

are matrix coefficients defining degree of coupling strength at a particular point $z$ in the structure between $m$ and $n$ modes.

For convenience we may rewriting (19) in a matrix form.

$$
\frac{d}{d z} \vec{c}(z)=D(z) \vec{c}(z)
$$

Considering the initial conditions we can solve the above system of coupled differential equations if we know all coupling coefficients along the $z$ axis of mode propagation. 


\subsection{Bragg Gratings Spectrum Calculation}

Let us examine an uniform Bragg grating in a single mode fibre. Simple solution for the reflection and transmission spectrum of FBG might be obtained when the coupling occurs between only two core modes. We can rewrite (19) for this case in the following form

$$
\frac{d}{d z}\left(\begin{array}{l}
c_{1}(z) \\
c_{2}(z)
\end{array}\right)=-\frac{i}{2} \omega^{2} \mu\left[\begin{array}{cc}
\frac{d_{11}(z)}{\beta_{1}} e^{-i\left(\beta_{1}-\beta_{1}\right) z} & \frac{d_{12}(z)}{\beta_{1}} e^{-i\left(\beta_{2}-\beta_{1}\right) z} \\
\frac{d_{21}(z)}{\beta_{2}} e^{-i\left(\beta_{1}-\beta_{2}\right) z} & \frac{d_{22}(z)}{\beta_{2}} e^{-i\left(\beta_{2}-\beta_{2}\right) z}
\end{array}\right]\left(\begin{array}{c}
c_{1}(z) \\
c_{2}(z)
\end{array}\right)
$$

To find matrix elements $d_{m n}$ we will consider a special case when the dielectric permittivity $\epsilon$ is a function of $z$ only with no dependency on $x$ and $y$ coordinates. Assuming such grating with only harmonic perturbation along $\mathrm{z}$ axis and the period $\Lambda$

$$
n(z)=n_{0}+\Delta n \cos \left(k_{B} z\right)
$$

where $k_{B}=\frac{2 \pi}{\Lambda}$ is the Bragg grating wave number.

$$
\epsilon(z)=\epsilon_{0}\left(n_{0}+\Delta n \cos \left(k_{B} z\right)\right)^{2} \simeq \epsilon_{0} n_{0}^{2}+\Delta \epsilon(z)
$$

Where $\Delta \epsilon(z)=\simeq \epsilon_{0} 2 n_{0} \Delta n * \cos \left(k_{B} z\right)$ is the perturbation along $\mathrm{z}$ axis.

Considering (20) we can rewrite matrix elements $d_{m n}(22)$ in the following form

$$
\begin{aligned}
& d_{12}(z)=2 \epsilon_{0} n_{0} \Delta n * \cos \left(k_{B} z\right) * \Gamma \\
& d_{21}(z)=2 \epsilon_{0} n_{0} \Delta n * \cos \left(k_{B} z\right) * \Gamma^{*} \\
& d_{11}(z)=d_{22}(z)=2 \epsilon_{0} n \Delta n \cos \left(k_{B} z\right)
\end{aligned}
$$




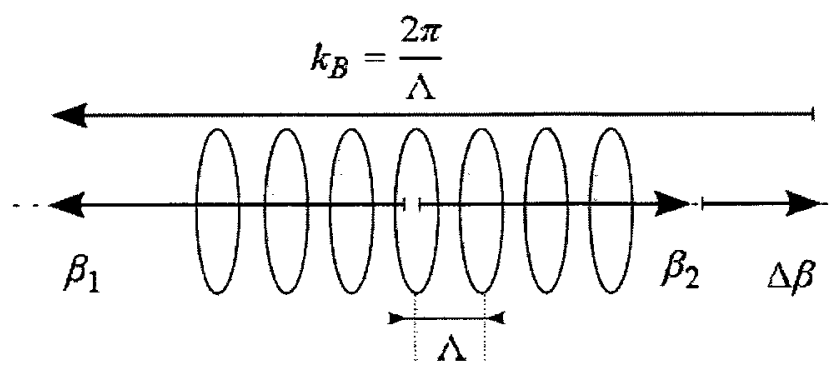

Figure 6: Bragg grating momentum diagram of contra-directional coupling for the general case of phase mismatch

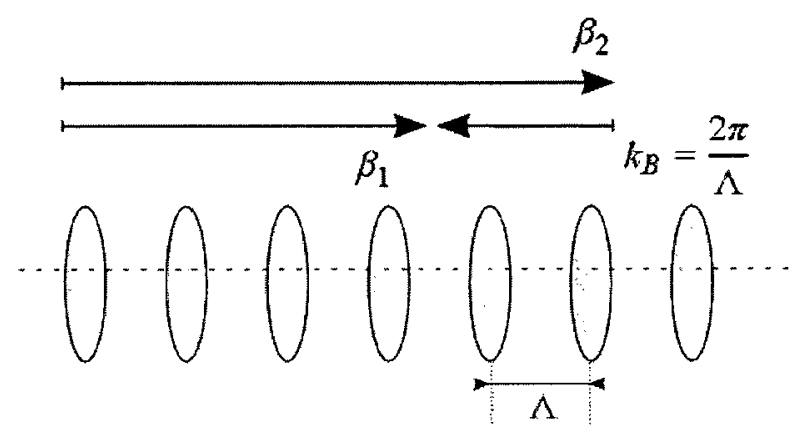

Figure 7: Long period Bragg grating momentum diagram of co-directional coupling

$$
\text { where } \Gamma=\frac{\int \overrightarrow{E_{1}} \overrightarrow{E_{2}^{*}} d x d y}{\int \overrightarrow{E_{1}} \overrightarrow{E_{2}^{*}} d x d y}
$$

Modes coupling can be either co-directional or contra-directional. Bragg gratings in which coupling occurs between modes traveling in opposite directions (contradirectional coupling) called reflection gratings or short-period gratings FBG (Figure 6). If coupling occurs between modes traveling in the same direction, gratings are called transmission gratings or long-period gratings LPBG (Figure 7). Both types of gratings are identical except the periods of Bragg gratings $\left(\sim 10^{-6}\right.$ meters FBG versus $\sim 10^{-4}$ meters for $\mathrm{LPBG}$ )

We will consider the case when we have a contra-directional coupling (forward and backward propagating modes) $\beta_{1} \beta_{2}<0$ with a small phase mismatch $\beta_{2}-\beta_{1} \neq 0$ (Figure 6). 
Considering our matrix elements (25) and momentum condition we can rewrite $(22)[16,17]$.

$$
\begin{aligned}
\frac{d}{d z} c_{1}(z) & =-i \gamma c_{2}(z) e^{j\left(\beta_{2}-\beta_{1}-k_{B}\right) z} \\
\frac{d}{d z} c_{2}(z) & =-i \gamma^{*} c_{2}(z) e^{-j\left(\beta_{2}-\beta_{1}-k_{B}\right) z}
\end{aligned}
$$

where $\gamma=\frac{1}{2} \frac{k_{0}^{2} n_{0} \Delta n}{\sqrt{\beta_{1} \beta_{2}}}$ is the coupling coefficient between forward and backward propagating modes [16],

$c_{1}(z), c_{2}(z)$ are the complex amplitudes of the normalized modes.

These are coupled first-order ordinary differential equations governing the interaction between two counter-propagating modes. We assume that we have the following boundary conditions 1) the forward propagating wave has unitary amplitude at the grating beginning $c_{1}(0)=1$, and 2) no backward-going wave exists at the end of the grating $c_{2}(L)=0$.

Solving (26) we can find Bragg grating spectral properties, such as reflectivity (Figure 8).

$$
R=\frac{\left|c_{2}(0)\right|^{2}}{\left|c_{1}(0)\right|^{2}}=\frac{\gamma^{2} \sinh ^{2}(\sigma L)}{\sigma^{2} \cosh ^{2}(\sigma L)-\left(\frac{\Delta \beta}{2}\right)^{2} \sinh ^{2}(\sigma L)} .
$$

where $\sigma^{2}=\gamma^{2}-{\frac{\Delta \beta^{2}}{2}}^{2}$

$\Delta \beta=\beta_{2}-\beta_{1}-\frac{2 \pi}{\Lambda}$ is the phase mismatch,

$L=N \Lambda$ is the grating length,

$N$ is the number of the grating periods.

We can see that a uniform Bragg grating acts as a reflectance filters by coupling energy away from the incident mode to the backward propagating mode. The 


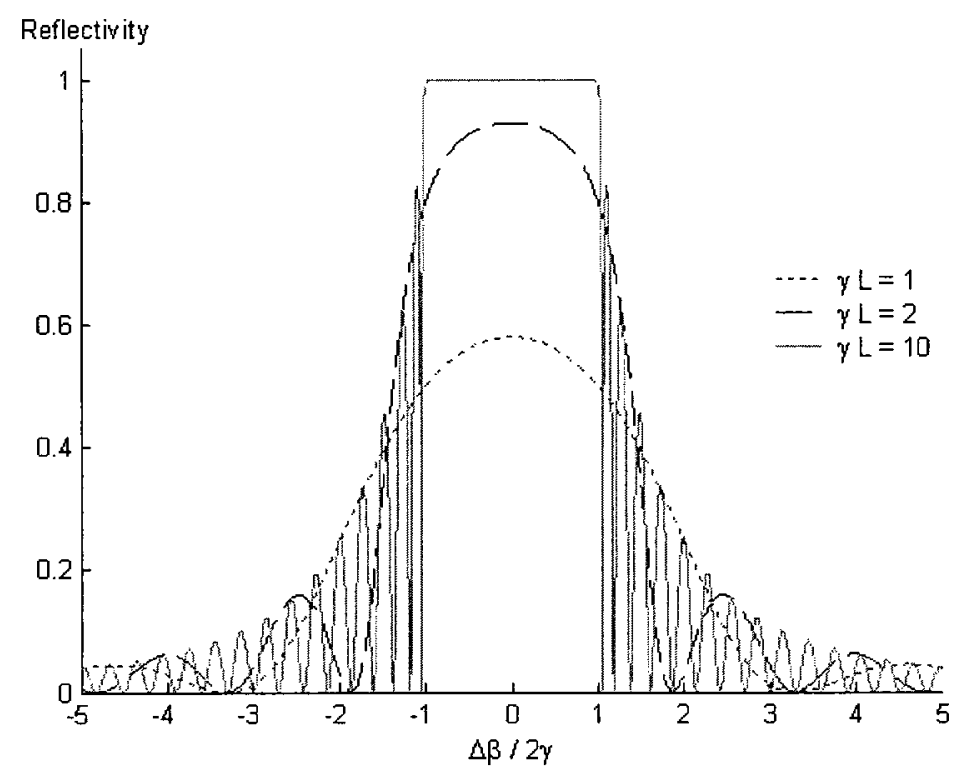

Figure 8: The reflectivity of a uniform grating as a function of phase mismatch.

bandwidth is determined by the coupling coefficient $\gamma$ and grating length $L$. The reflection maximum occurs at the Bragg wavelength when phase mismatch $\Delta \beta$ is zero and $\beta_{2}=-\beta_{1}$, i.e. forward and backward propagating modes have opposite momentum.

Transmission spectrum of a grating simply might be obtained from energy conservation law $T=1-R$. At the Bragg wavelength we will observe a sharp dip in the transmission spectrum.

Other cases e.g. coupling from forward propagating core mode to a backward propagating cladding mode will be discussed in section 2.5 . 


\section{$2.4 \quad$ Bragg Grating Types}

The spectrum of FBG is determined by the grating period function and grating shape. Filters of particular shapes with narrow bandwidth are required for passive components in dense wavelength-division multiplexed (DWDM) communications systems.

There are six common structures for FBGs [17] as shown un Figure 9.

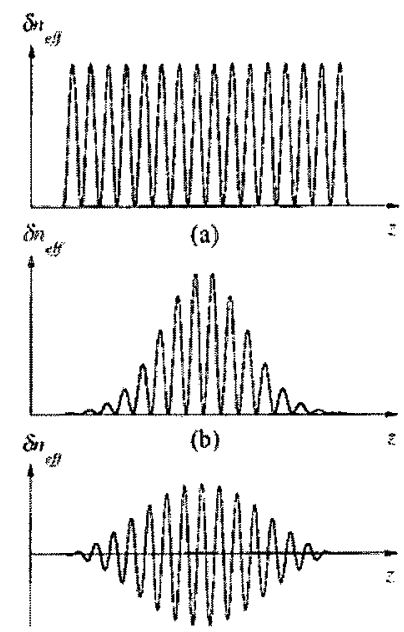

(c)

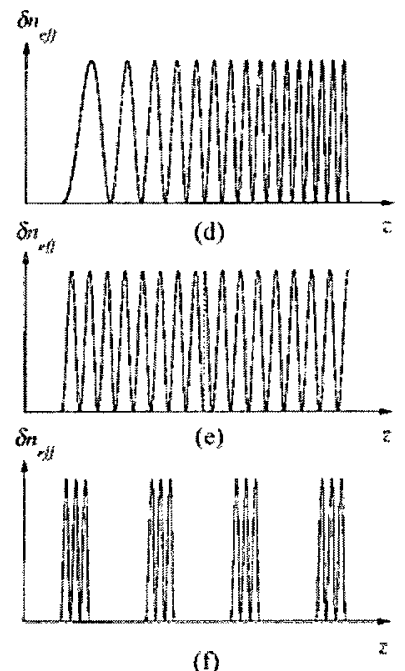

(f)

Figure 9: Fibre gratings types:

(a) uniform with positive-only index change, (b) Gaussian-apodized,

(c) raised-cosine-apodized with zero-dc index change, (d) chirped,

(e) discrete phase shift, (f) superstructure.

For some applications a nonuniform grating is required to reduce the undesirable side lobes appeared in uniform grating spectra (Figure 8). Apodized gratings offer significant improvement in side-lobe suppression while maintaining a narrow bandwidth. The two functions typically used to apodize a FBG are Gaussian (Figure 9 b) and raised-cosine (Figure $9 \mathrm{c}$ ).

The grating can be modified to add other features, such as a linear variation in the grating period, called a chirp, to add dispersion to the grating response (Figure $9 \mathrm{~d}$ ). Chirped FBG are used for polarization and dispersion compensation, controlling and shaping short pulses in fibre lasers [18], [19]. The grating period can be and either 
localised or distributed in a superstructure (Figure $9 \mathrm{f}$ ).

In the previous Chapter we have assumed that the index profile is sinusoidal, however in the general case the profile might be of any shape. To find a coupling coefficients for a general type of grating we can represent index profile function $\Delta \epsilon(x, y, z)$ as a Fourier series [20].

$$
\Delta \epsilon(x, y, z)=\sum_{p=0}^{\infty} \gamma_{p}(x, y) e^{-i p \frac{2 \pi}{\Lambda} z}
$$

and

$$
\gamma_{p}(x, y)=\frac{2}{\Lambda} \int_{-\frac{\Lambda}{2}}^{\frac{\Lambda}{2}} \Delta \epsilon(x, y, z) e^{i p \frac{2 \pi}{\Lambda} z} d z
$$

Fourier coefficients $\gamma_{p}$ represent given structure perturbated along the $z$ axis. We can rewrite (19)

$$
\frac{d c_{n}(z)}{d z}=-\frac{i}{2} \frac{\omega^{2} \mu}{\beta_{n}} \sum_{m, p} c_{m}(z) d_{m n}^{p} e^{-i\left(\beta_{m}-\beta_{n}+p \frac{2 \pi}{\Lambda}\right) z}
$$

With matrix coefficients calculated for each Fourier term separately.

$$
d_{m n}^{p}=\frac{\int \gamma_{p}(x, y) \overrightarrow{E_{m}} \overrightarrow{E_{n}^{*}} d x d y}{\int \overrightarrow{E_{n}} \overrightarrow{E_{n}^{*}} d x d y}
$$




\subsection{Mode Coupling in a Tilted Fibre Bragg Grat- ing}

A Fibre Bragg grating with a tilt of the grating fringes is called tilted fibre Bragg grating (TFBG) (Figure 10). The tilted grating causes coupling between core and cladding modes. For a big tilt angle energy from core might be coupled to radiation modes as well.

We can write the perturbation of induced refractive index in the following form $[21,22]$.

$$
n(x, z)=n_{0}+\Delta n \cos \left(k_{B} \cos (\theta) z+k_{B} \sin (\theta) x\right)
$$

where $k_{B}=\frac{2 \pi}{\Lambda_{B}}$ is the Bragg grating wave number. Substituting (32) into we can write coupling coefficients in the following form.

$$
d_{m n}(\theta, z)=2 n_{c} \Delta n \frac{\int \cos \left(k_{B} \cos (\theta) z+k_{B} \sin (\theta) x\right) \overrightarrow{E_{m}} \vec{E}_{n}^{*} d x d y}{\int \overrightarrow{E_{n}} \overrightarrow{E_{n}^{*}} d x d y}
$$

As we can see our matrix coefficient are determined by the tilt angle $\theta$ which results in different strength of mode coupling for different tilt angles.

To calculate a TFBG spectrum we have to take into consideration not only coupling coefficients between core and cladding modes, but find all coupling coefficients between modes, as energy might be transfered between cladding-guided modes. In

the general case if the cladding support $n$ modes we need to find $\frac{1}{2} n \times n$ coefficients, i.e. overlap integrals between mode profiles.

Figure 11 illustrates the measured coupling of a core mode cladding modes. The large transmission dip is the Bragg reflection resonance, while the remaining dips are related to the coupling to cladding modes. 


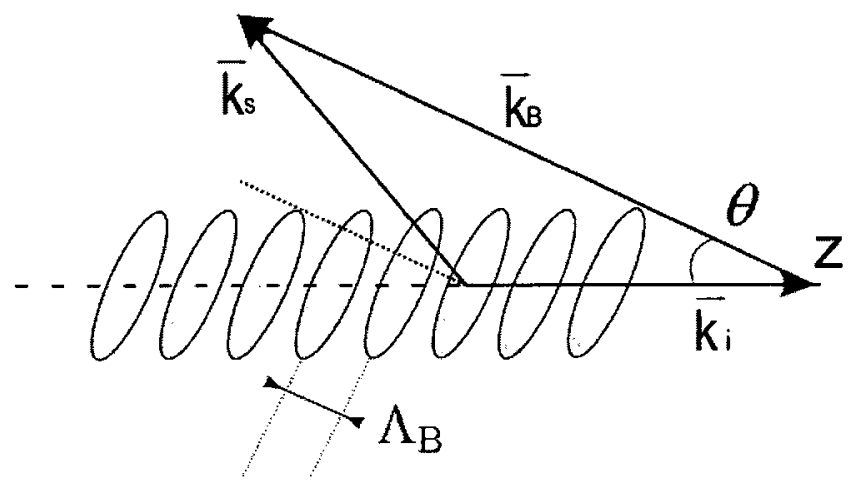

Figure 10: TFBG momentum vector diagram of contra-directional coupling.

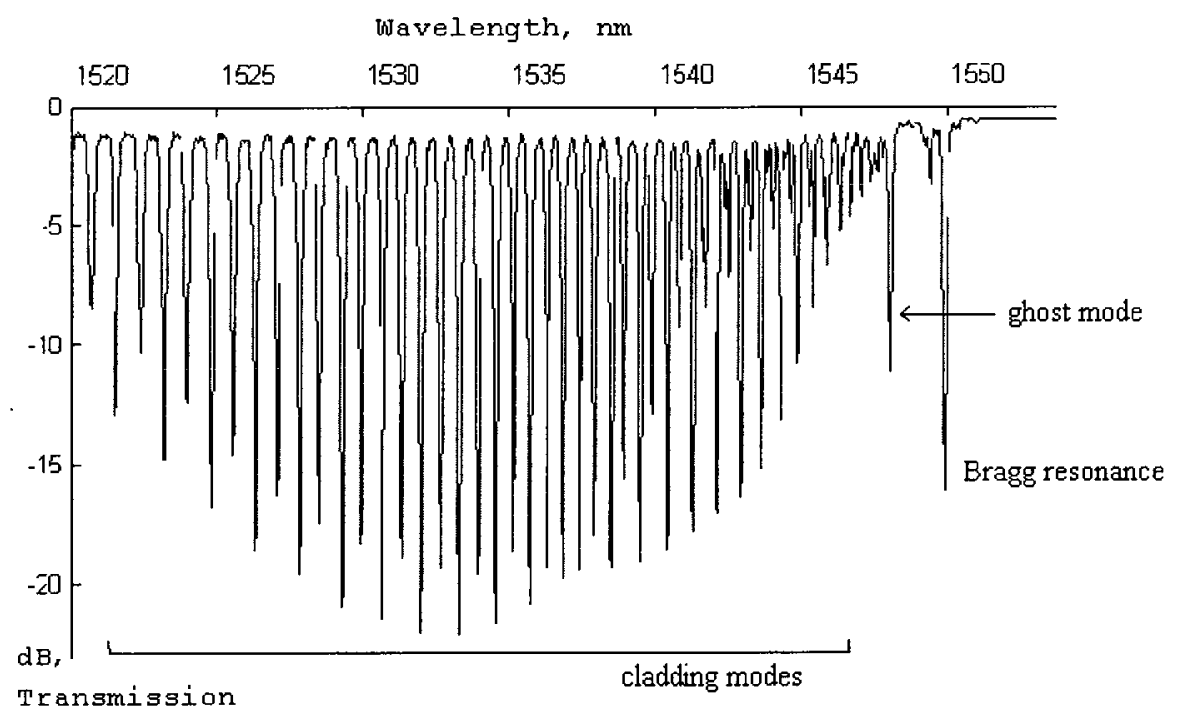

Figure 11: Typical TFBG spectrum $\left(\theta=6^{0}\right)$ 
An important feature of the weakly (2..10 degrees ) tilted FBGs is the presence of a strong "ghost mode" resonance, immediately to the left of the Bragg resonance in Figure 11. The ghost resonance corresponds to a group of nearly degenerate low-order asymmetric cladding modes which are well confined in the core and less sensitive to the cladding-external medium interface [23]. 


\section{Chapter 3}

\section{Optical Modes in a Multilayer Step-Index Cylindrical Waveguide}

\subsection{Electromagnetic Fields in Cylindrical Waveguides}

In order to calculate coupling coefficients between modes in a multilayer cylindrical structure i.e. FBG, the modes and propogation constants should be determined first.

The problem of interest includes a metal coating on the fibre surface which results in a significant refractive index variation (Figure 12). In this case we have to use vector analysis to consider coupling between magnetic and electric field accrued at the boundary. This approach will lead us to the hybrid modes analysis of the fibre.

We will derive a general equation suitable for any number of layers with any kind of refractive index. In this work we are interested in a 4 layer fibre cylindrical structure with a big refractive index variation between the layers. Examples for one and two layer systems with small refractive index variation can be found in [24-30]. 


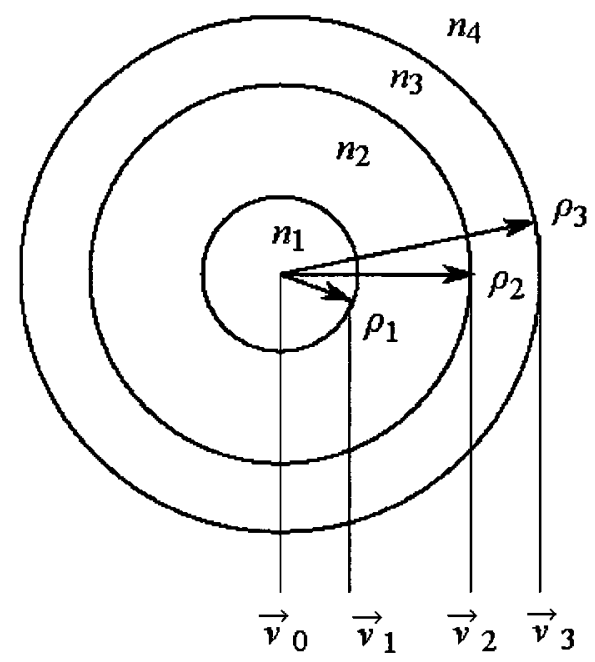

Figure 12: Schematic structure of 4 layer circular waveguide

We will start from source free Maxwell's equations:

$$
\begin{aligned}
\nabla \times \vec{E} & =-\partial_{t} \vec{B}, \\
\nabla \times \vec{H} & =\partial_{t} \vec{D}, \\
\nabla * \vec{B} & =0, \\
\nabla * \vec{D} & =0 .
\end{aligned}
$$

The role of medium in the interaction with an electromagnetic field is described by the following two equations

$$
\begin{gathered}
\vec{D}=\epsilon_{0} \vec{E}+\vec{P}=\epsilon_{0} \epsilon_{r} \vec{E} . \\
\vec{B}=\mu_{0} \mu_{r} \vec{H} .
\end{gathered}
$$

For nonmagnetic materials: $\vec{B}=\mu_{0} \vec{H}$.

Assuming harmonic fields $\psi(\vec{r}, t)=\psi(\vec{r}) e^{j \omega t}$ we can rewrite Maxwell's equations 
in the frequency domain

$$
\begin{aligned}
\nabla \times \vec{H} & =-j \omega \epsilon_{0} \epsilon_{r} \vec{E}, \\
\nabla \times \vec{E} & =j \omega \mu_{0} \vec{H}, \\
\nabla * \vec{H} & =0, \\
\nabla *\left(\epsilon_{r} \vec{E}\right) & =0 .
\end{aligned}
$$

Considering a cylindrical symmetry of the problem we can rewrite Maxwell's equations

$$
\nabla \times \vec{H}=-j \omega \epsilon \vec{E}
$$

where operator $\nabla$ in cylindrical coordinates has representation

$$
\nabla=\hat{\rho} \partial_{\rho}+\hat{\theta} \frac{1}{\rho} \partial_{\theta}+\hat{z} \partial_{z}=\left(\begin{array}{lll}
\hat{\rho} & \hat{\theta} & \hat{z}
\end{array}\right)\left(\begin{array}{c}
\partial_{\rho} \\
\frac{1}{\rho} \partial_{\theta} \\
\partial_{z}
\end{array}\right)
$$


Therefore

$$
\begin{aligned}
\nabla \times \vec{H}=\operatorname{det}\left[\begin{array}{ccc}
\hat{\rho} & \hat{\theta} & \hat{z} \\
\partial_{\rho} & \frac{1}{\rho} \partial_{\theta} & \partial_{z} \\
H_{r} & H_{\theta} & H_{z}
\end{array}\right]=\operatorname{det}\left[\begin{array}{ccc}
\frac{\hat{\rho}}{\rho} & \hat{\theta} & \frac{\hat{z}}{\rho} \\
\partial_{\rho} & \partial_{\theta} & \partial_{z} \\
H_{r} & \rho H_{\theta} & H_{z}
\end{array}\right]= \\
= \\
=\left(\begin{array}{c}
\frac{1}{\rho} \partial_{\theta} H_{z}-\partial_{z} H_{\theta} \\
\partial_{z} H_{r}-\partial_{\rho} H_{z} \\
\frac{1}{\rho} \partial_{\rho}\left(\rho H_{\theta}\right)-\frac{1}{\rho} \partial_{\theta} H_{\rho}
\end{array}\right)=\left(\begin{array}{c}
\frac{1}{\rho} \partial_{\theta} H_{z}-j \beta H_{\theta} \\
j \beta H_{r}-\partial_{\rho} H_{z} \\
\frac{1}{\rho} \partial_{\rho}\left(\rho H_{\theta}\right)-\frac{1}{\rho} \partial_{\theta} H_{\rho}
\end{array}\right)= \\
=-j \omega \epsilon\left(\begin{array}{c}
E_{\rho} \\
E_{\theta} \\
E_{z}
\end{array}\right) .
\end{aligned}
$$

The latter result was obtained with the use of determinant properties. The second column was multiplying by $\rho$ and the first row was dividing by $\rho$ without affecting the value of the determinant.

We have also assumed uniformity of the fibre structure along $z$ axis, which results in a periodic field with respect to the $z$ coordinate $e^{j \beta z}$ and leading to the replacement of $\partial_{z}$ with $j \beta$ (since we are looking for modes with a well defined propagating constant $\beta)$.

In the same manner we can rewrite (41)

$$
\nabla \times \vec{E}=j \omega \mu \vec{H}
$$




$$
\nabla \times \vec{E}=\left(\begin{array}{c}
\frac{1}{\rho} \partial_{\theta} E_{z}-j \beta E_{\theta} \\
j \beta E_{r}-\partial_{\rho} E_{z} \\
\frac{1}{\rho} \partial_{\rho}\left(\rho E_{\theta}\right)-\frac{1}{\rho} \partial_{\theta} E_{\rho}
\end{array}\right)=j \omega \mu\left(\begin{array}{c}
H_{\rho} \\
H_{\theta} \\
H_{z}
\end{array}\right)
$$

We can rewrite latter 6 equations (46) and (48) in a slightly different form

$$
\begin{aligned}
& E_{\rho}=\frac{j}{\gamma^{2}}\left(\beta \partial_{\rho} E_{z}+\omega \mu \frac{1}{\rho} \partial_{\theta} H_{z}\right) \\
& E_{\theta}=\frac{j}{\gamma^{2}}\left(\frac{\beta}{\rho} \partial_{\theta} E_{z}-\omega \mu \partial_{\rho} H_{z}\right) \\
& H_{\rho}=\frac{j}{\gamma^{2}}\left(-\omega \epsilon \frac{1}{\rho} \partial_{\theta} E_{z}+\beta \partial_{\rho} H_{z}\right), \\
& H_{\theta}=\frac{j}{\gamma^{2}}\left(\omega \epsilon \partial_{\rho} E_{z}+\frac{\beta}{\rho} \partial_{\theta} H_{z}\right),
\end{aligned}
$$

and isolate $E_{z}$ and $H_{z}$ as

$$
\begin{aligned}
& \left(\partial_{\rho}^{2}+\frac{1}{\rho} \partial_{\rho}+\frac{1}{\rho^{2}} \partial_{\theta}^{2}+\gamma^{2}\right) E_{z}=0 \\
& \left(\partial_{\rho}^{2}+\frac{1}{\rho} \partial_{\rho}+\frac{1}{\rho^{2}} \partial_{\theta}^{2}+\gamma^{2}\right) H_{z}=0
\end{aligned}
$$

We can rewrite latter two equation in a simple form

$$
\left(\nabla_{T}^{2}+\gamma_{i}^{2}\right)\left(\begin{array}{c}
E_{z} \\
H_{z}
\end{array}\right)=0
$$

Where

$\nabla_{T}^{2}=\partial_{\rho}^{2}+\frac{1}{\rho} \partial_{\rho}+\frac{1}{\rho^{2}} \partial_{\theta}^{2}$ is the transverse part of the Laplace operator.

$\gamma_{i}^{2}=n_{i}^{2} k_{0}^{2}-\beta^{2}$ is the transverse propagation constant in the $i$ th layer of the 
multilayer medium, for a wave with propagating constant $\beta$ along $\hat{z}$

Now we can solve two scalar equations (53) and (54) and substitute $E_{z}, H_{z}$ into (49)- (52) to obtain $E_{\rho}, E_{\theta}, H_{\rho}, H_{\theta}$.

Let us take a look at general solution of (53), (54).

$$
\left(d_{\rho}^{2}+\frac{1}{\rho} d_{\rho}+\frac{1}{\rho^{2}} \partial_{\theta}^{2}+\gamma^{2}\right) \psi(\rho, \theta)=0
$$

where we have further removed the $\mathrm{z}$ dependency of $\psi(\rho, \theta, z)$ by using

$$
\psi(\rho, \theta, z)=\psi(\rho, \theta) e^{-j \beta z}
$$

Assuming angular periodicity for a cylindrical structure along the $\hat{\theta}$ direction, and applying Fourier decomposition we get

$$
\begin{gathered}
\psi(\rho, \theta)=\sum_{m=-\infty}^{\infty} \psi_{m}(\rho) e^{j m \theta}, \\
\psi_{m}(\rho)=\frac{1}{2 \pi} \int_{-\pi}^{\pi} \psi(\rho, \theta) e^{-j m \theta} d \theta .
\end{gathered}
$$

Substituting (58) in (57) we get

$$
\begin{gathered}
\left(d_{\rho}^{2}+\frac{1}{\rho} d_{\rho}+\left(\gamma^{2}-\frac{m^{2}}{\rho^{2}}\right)\right) \psi_{m}(\rho)=0, \\
\left(\rho^{2} d_{\rho}^{2}+\rho d_{\rho}+\left((\rho \gamma)^{2}-m^{2}\right)\right) \psi_{m}(\rho)=0,
\end{gathered}
$$

replacing

$x:=\gamma \rho, \rho \frac{d}{d \rho}=\rho \gamma \frac{d}{\gamma d \rho}=x \frac{d}{d x}, \rho^{2} \frac{d^{2}}{d \rho^{2}}=x^{2} \frac{d^{2}}{d x^{2}}$ and $\psi_{m}(\rho) \rightarrow y_{m}(x)$ we get

$$
x^{2} y^{\prime \prime}+x y^{\prime}+\left(x^{2}-m^{2}\right) y=0 .
$$


Equation (62) is a $m$ th order Bessel equation. In every $i$ th uniform region of Figure 12 the general solutions to the Bessel equation are Bessel functions of order $m$.

For each layer characterized by its refractive index $n_{i}$, we have a set of two independent solutions for two possible cases:

1) for $n_{i}^{2} k_{0}^{2}>\beta^{2}$ we have $\gamma_{i}=\sqrt{n_{i}^{2} k_{0}^{2}-\beta^{2}}$ real, $x=\rho \sqrt{n_{i}^{2} k_{0}^{2}-\beta^{2}}$ is real, then

$$
y_{m}(x)=c_{1} J_{m}(x)+c_{2} Y_{m}(x)
$$

2) for $n_{i}^{2} k_{0}^{2}<\beta^{2}$ we have $\gamma_{i}=i \sqrt{\beta^{2}-n_{i}^{2} k_{0}^{2}}$ imaginary, $x=\rho \sqrt{\beta^{2}-n_{i}^{2} k_{0}^{2}}$ is real

$$
\begin{aligned}
y_{m}(x) & =c_{1} J_{m}(i x)+c_{2} Y_{m}(i x)= \\
& =c_{1}^{\prime} I_{m}(x)+c_{2}^{\prime} K_{m}(x) .
\end{aligned}
$$

Where $J_{m}$ and $Y_{m}$ are Bessel functions of the first and the second kind. $I_{m}$ and $K_{m}$ are modified Bessel functions of the first and the second kind.

Therefore we can conclude that for each $i$ th layer the solution of the Helmholtz equation (55)

$$
\left(d_{\rho}^{2}+\frac{1}{\rho} d_{\rho}+\left(\gamma_{i}^{2}-\frac{m^{2}}{\rho^{2}}\right)\right)\left(\begin{array}{c}
E_{z m}^{(i)}(\rho) \\
H_{z m}^{(i)}(\rho)
\end{array}\right)=0
$$

will give longitudinal components of electric and magnetic field as

$$
\begin{aligned}
& E_{m z}^{(i)}(\rho, \theta)=E_{z}(\rho) e^{-j m \theta}=\left(A_{m}^{(i)} J_{m}+B_{m}^{(i)} Y_{m}\right) e^{-j m \theta} \\
& H_{m z}^{(i)}(\rho, \theta)=H_{z}(\rho) e^{-j m \theta}=\left(C_{m}^{(i)} J_{m}+D_{m}^{(i)} Y_{m}\right) e^{-j m \theta}
\end{aligned}
$$




\subsection{Boundary conditions}

In order to find specific solutions for a given waveguide structure we need to apply boundary conditions. Any structure with cylindrical-symmetry might be represented as a sequence of cylindrical layers. By choosing each layer small enough we may consider it to have uniform properties. Our goal here is to find conditions on the boundary between these layers. The mode solutions in the whole region of interest can be found by combining fields from all such layers in such a way that all boundary conditions are satisfied.

Boundary condition yields continuity of the tangential components of electrical and magnetic field $\vec{E}$ and $\vec{H}$. Therefore in our case of cylindrical-symmetry field $E z$, $E_{\theta}$, and $H z, H_{\theta}$ should be continuous.

We can rewrite equations (66) in matrix form

$$
\left(\begin{array}{c}
E_{z} \\
H_{z}
\end{array}\right)=e^{-j m \theta}\left[\begin{array}{ll}
A_{m} & B_{m} \\
C_{m} & D_{m}
\end{array}\right]\left(\begin{array}{c}
J_{m}(\gamma \rho) \\
Y_{m}(\gamma \rho)
\end{array}\right) .
$$

To collect all unknown coefficients $A_{m}, B_{m}, C_{m}, D_{m}$ in one single vector we can rewrite the latter equation in the form

$$
\left(\begin{array}{c}
E_{z} \\
H_{z}
\end{array}\right)=e^{-j m \theta}\left[\begin{array}{cccc}
J_{m} & Y_{m} & 0 & 0 \\
0 & 0 & J_{m} & Y_{m}
\end{array}\right]\left(\begin{array}{c}
A_{m} \\
B_{m} \\
C_{m} \\
D_{m}
\end{array}\right)
$$

Now we can find angular components of an electromagnetic field from (50) and 
(52)

$$
\begin{aligned}
& E_{\theta}=\frac{j}{\gamma^{2}}\left(\frac{\beta}{\rho} \partial_{\theta} E_{z}-\omega \mu \partial_{\rho} H_{z}\right) \\
& H_{\theta}=\frac{j}{\gamma^{2}}\left(\frac{\beta}{\rho} \partial_{\theta} H_{z}+\omega \epsilon \partial_{\rho} E_{z}\right)
\end{aligned}
$$

Rewriting the latter equations in the matrix form

$$
\left(\begin{array}{c}
E_{\theta} \\
H_{\theta}
\end{array}\right)=\frac{j}{\gamma^{2}}\left(\frac{\beta}{\rho} \partial_{\theta}\left(\begin{array}{c}
E_{z} \\
H_{z}
\end{array}\right)+\omega\left[\begin{array}{cc}
0 & -\mu \\
\epsilon & 0
\end{array}\right] \partial_{\rho}\left(\begin{array}{l}
E_{z} \\
H_{z}
\end{array}\right)\right),
$$

where from (67) we know that

$$
\begin{aligned}
\partial_{\theta}\left(\begin{array}{c}
E_{z} \\
H_{z}
\end{array}\right) & =-j m e^{-j m \theta}\left[\begin{array}{ll}
A_{m} & B_{m} \\
C_{m} & D_{m}
\end{array}\right]\left(\begin{array}{l}
J_{m} \\
Y_{m}
\end{array}\right), \\
\partial_{\rho}\left(\begin{array}{l}
E_{z} \\
H_{z}
\end{array}\right) & =\gamma e^{-j m \theta}\left[\begin{array}{ll}
A_{m} & B_{m} \\
C_{m} & D_{m}
\end{array}\right]\left(\begin{array}{l}
J_{m}^{\prime} \\
Y_{m}^{\prime}
\end{array}\right) .
\end{aligned}
$$

Substituting these expressions in (71) we get

$$
\begin{aligned}
\left(\begin{array}{c}
E_{\theta} \\
H_{\theta}
\end{array}\right)= & \frac{j}{\gamma^{2}} e^{-j m \theta}\left(-j \frac{\beta}{\rho} m\left[\begin{array}{ll}
A_{m} & B_{m} \\
C_{m} & D_{m}
\end{array}\right]\left(\begin{array}{c}
J_{m} \\
Y_{m}
\end{array}\right)+\right. \\
& \left.+\omega \gamma\left[\begin{array}{cc}
0 & -\mu \\
\epsilon & 0
\end{array}\right]\left[\begin{array}{ll}
A_{m} & B_{m} \\
C_{m} & D_{m}
\end{array}\right]\left(\begin{array}{c}
J_{m}^{\prime} \\
Y_{m}^{\prime}
\end{array}\right)\right)
\end{aligned}
$$


or

$$
\left(\begin{array}{c}
E_{\theta} \\
H_{\theta}
\end{array}\right)=e^{-j m \theta}\left[\begin{array}{cccc}
\frac{\beta}{\rho \gamma^{2}} m J_{m} & \frac{\beta}{\rho \gamma^{2}} m Y_{m} & -j \mu \frac{\omega}{\gamma} J_{m}^{\prime} & -j \mu \frac{\omega}{\gamma} Y_{m}^{\prime} \\
j \epsilon \frac{\omega}{\gamma} J_{m}^{\prime} & j \epsilon \frac{\omega}{\gamma} \gamma Y_{m}^{\prime} & \frac{\beta}{\rho \gamma^{2}} m J_{m} & \frac{\beta}{\rho \gamma^{2}} m Y_{m}
\end{array}\right]\left(\begin{array}{c}
A_{m} \\
B_{m} \\
C_{m} \\
D_{m}
\end{array}\right)
$$

Finally combining equations (68) and (75) we get

$$
\begin{aligned}
&\left.\left.\left(\begin{array}{c}
E_{z} \\
E_{\theta} \\
H_{z} \\
H_{\theta}
\end{array}\right]=\left[\begin{array}{cccc}
J_{m} & Y_{m} & 0 & 0 \\
\frac{\beta}{\rho \gamma^{2}} m J_{m} & \frac{\beta}{\rho \gamma^{2}} m Y_{m} & -j \mu \frac{\omega}{\gamma} J_{m}^{\prime} & -j \mu \frac{\omega}{\gamma} Y_{m}^{\prime} \\
0 & 0 & J_{m} & Y_{m} \\
j \epsilon \frac{\omega}{\gamma} J_{m}^{\prime} & j \epsilon \frac{\omega}{\gamma} \gamma Y_{m}^{\prime} & \frac{\beta}{\rho \gamma^{2}} m J_{m} & \frac{\beta}{\rho \gamma^{2}} m Y_{m}
\end{array}\right]\right) \begin{array}{c}
A_{m} \\
C_{m} \\
B_{m} \\
D_{m}
\end{array}\right)= \\
&= {\left[\begin{array}{cc}
m^{E} & m^{E H} \\
m^{H E} & m^{H}
\end{array}\right]\left(\begin{array}{c}
A_{m} \\
B_{m} \\
C_{m} \\
D_{m}
\end{array}\right)=\left[\begin{array}{cc}
a & b \\
-\frac{\epsilon}{\mu} b & a
\end{array}\right]\left(\begin{array}{c}
A_{m} \\
C_{m} \\
B_{m} \\
D_{m}
\end{array}\right) . }
\end{aligned}
$$


Or simply rewriting the last equation for an arbitrary $i$ th layer we get

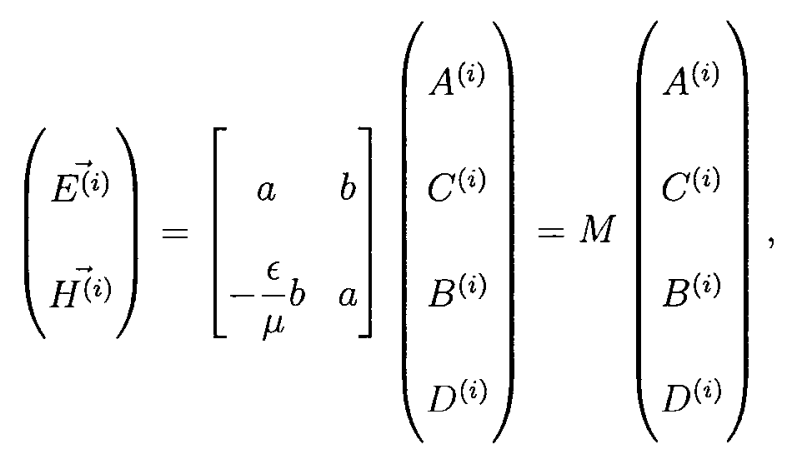

where

$$
\begin{aligned}
a=m^{E}=m^{H} & =\left[\begin{array}{cc}
J_{m} & Y_{m} \\
\frac{\beta}{\rho \gamma^{2}} m J_{m} & \frac{\beta}{\rho \gamma^{2}} m Y_{m}
\end{array}\right], \\
b=m^{E H}=-\frac{\epsilon}{\mu} m^{H E} & =\left[\begin{array}{cc}
0 & 0 \\
-j \mu \frac{\omega}{\gamma} J_{m}^{\prime} & -j \mu \frac{\omega}{\gamma} Y_{m}^{\prime}
\end{array}\right] .
\end{aligned}
$$

Matrix $b=m^{E H}$ describes coupling between electric $\vec{E}$ and magnetic field $\vec{H}$ on the boundary, which is true only for the general case vectorial wave approach.

Where

$M=M(m, \omega, \rho, \gamma)$ is the transfer matrix for $i$ th layer,

$\gamma=\sqrt{\left(n k_{0}\right)^{2}-\beta^{2}}$ is the mode wave number,

$\beta$ is the mode propagation wave number along the $z$ axis,

$k_{o}=\omega / c$ is the the free space wave number, $m$ is the mode angular symmetry number, $A, B, C, D$ are unknown coefficients for some $i$ th layer for $m$ mode number and propagating constant $\beta$. 


\subsection{Matrix method}

In order to find propagation constant and mode profiles we can use matrix method [24,29-31]. With the help of matrix equation we can connect field components from the opposite sides of each uniform layer. With help of boundary conditions we can connect the field matrices $M^{(i)}$ for all layers.

Let us denote $\vec{\psi}(\rho)=\left(\begin{array}{c}E_{z}(\rho) \\ E_{\theta}(\rho) \\ H_{z}(\rho) \\ H_{\theta}(\rho)\end{array}\right)$ and $\overrightarrow{v_{i}}=\left(\begin{array}{c}A_{i} \\ C_{i} \\ B_{i} \\ D_{i}\end{array}\right)$, see Figure 12 .

We can write boundary condition in the following form

$$
\vec{\psi}\left(\rho_{i}-0\right)=\vec{\psi}\left(\rho_{i}+0\right)
$$

The field at the first boundary $\rho_{1}$ is defined by equation

$$
\vec{\psi}\left(\rho_{1}-0\right)=M_{1}\left(\rho_{1}\right) \overrightarrow{v_{0}}
$$

Applying boundary condition at $\rho_{1}$ we get

$$
\vec{\psi}\left(\rho_{1}+0\right)=\vec{\psi}\left(\rho_{1}-0\right)=M_{1}\left(\rho_{1}\right) \overrightarrow{v_{0}}
$$

When the field at the second layer close to the first boundary is

$$
\vec{\psi}\left(\rho_{1}+0\right)=M_{2}\left(\rho_{1}\right) \overrightarrow{v_{1}}
$$


Combining (81) and (83) we get

$$
M_{2}\left(\rho_{1}\right) \overrightarrow{v_{1}}=M_{1}\left(\rho_{1}\right) \overrightarrow{v_{0}}
$$

or

$$
\overrightarrow{v_{1}}=M_{2}^{-1}\left(\rho_{1}\right) M_{1}\left(\rho_{1}\right) \overrightarrow{v_{0}}
$$

In the similar way we can find field at the remaining boundaries

$$
\begin{gathered}
\vec{\psi}\left(\rho_{2}-0\right)=M_{2}\left(\rho_{2}\right) \overrightarrow{v_{1}}=M_{2}\left(\rho_{2}\right) M_{2}^{-1}\left(\rho_{1}\right) M_{1}\left(\rho_{1}\right) \overrightarrow{v_{0}} \\
\vec{\psi}\left(\rho_{2}+0\right)=M_{3}\left(\rho_{2}\right) \overrightarrow{v_{2}} \\
\overrightarrow{v_{2}}=M_{3}^{-1}\left(\rho_{2}\right) M_{2}\left(\rho_{2}\right) M_{2}^{-1}\left(\rho_{1}\right) M_{1}\left(\rho_{1}\right) \overrightarrow{v_{0}} \\
\overrightarrow{v_{3}}=M_{4}^{-1}\left(\rho_{3}\right) M_{3}\left(\rho_{3}\right) M_{3}^{-1}\left(\rho_{2}\right)^{-1} M_{2}\left(\rho_{2}\right) M_{2}^{-1}\left(\rho_{1}\right) M_{1}\left(\rho_{1}\right) \overrightarrow{v_{0}}
\end{gathered}
$$

or simply

$$
\left(\begin{array}{l}
A_{4} \\
C_{4} \\
B_{4} \\
D_{4}
\end{array}\right)=M\left(\begin{array}{l}
A_{0} \\
C_{0} \\
B_{0} \\
D_{0}
\end{array}\right) .
$$

Where

$$
M=\Pi_{i=1}^{3} M_{i+1}^{-1}\left(\rho_{i}\right) M_{i}\left(\rho_{i}\right)
$$

Note that the inverse matrices $M_{i}^{-1}$ can be calculated symbolically if they are ill defined.

We have 4 equations (90), but 8 unknown parameters. The remaining four conditions can be added if physical constrains are taken into consideration (no infinite 
fields): We could note that for some cases the second solution is unphysical:

1) for $\rho=0$ the Bessel function $Y$ will approach infinity $Y(0) \rightarrow \infty$.

2) for the $\rho \rightarrow \infty$ we have $I(\infty) \rightarrow \infty$

Therefore we should equate coefficients $B_{0}, D_{0}, A_{4}, C_{4}$ to zero.

$$
\left(\begin{array}{c}
0 \\
0 \\
B_{4} \\
D_{4}
\end{array}\right)=M\left(\begin{array}{l}
A_{0} \\
C_{0} \\
0 \\
0
\end{array}\right)=\left[\begin{array}{ll}
m_{11} & m_{12} \\
m_{21} & m_{22}
\end{array}\right]\left(\begin{array}{c}
A_{0} \\
C_{0} \\
0 \\
0
\end{array}\right)
$$

or

$$
\begin{aligned}
& \left(\begin{array}{l}
0 \\
0
\end{array}\right)=m_{11}\left(\begin{array}{l}
A_{0} \\
C_{0}
\end{array}\right), \\
& \left(\begin{array}{l}
B_{4} \\
D_{4}
\end{array}\right)=m_{21}\left(\begin{array}{l}
A_{0} \\
C_{0}
\end{array}\right) .
\end{aligned}
$$

We are interested in (93), as it allows us to find propagation mode constant. Indeed by taking determinant from both sides we get

$$
\operatorname{det}\left(m_{11}\right)=M_{11} M_{12}-M_{21} M_{12}=0
$$

Where $m_{11}=\left[\begin{array}{cc}M_{11} & M_{12} \\ M_{21} & M_{22}\end{array}\right]$ is $2 \times 2$ matrix constructed from the elements of matrix $M$, that depends only on the fibre parameters $\gamma_{1,2,3,4}, \rho_{1,2,3}, \epsilon_{1,2,3,4}$, angular symmetry number $m$ and one variable $\beta$, the mode propagation constant, which is found by 
solving (95). 


\section{Chapter 4}

\section{Numerical Simulation}

\subsection{Introduction}

The objective of this Section is to simulate a multilayer cylindrical structure with an arbitrary index profile function, to determine all possible mode propagation constants $\beta$ and correspondent mode profiles.

In each layer of the multilayer medium we can describe electromagnetic field with two Helmholtz equations for $\vec{E}$ and $\vec{H}$ separately (65). When the refractive index function of the structure varies slowly, we can consider these equations to be independent, thus we need to solve just one scalar equation. This approach is called the linear polarization modes approximation, and is used in this chapter.

The primary goal was find numerical solution of the eigenvalue equation (65) for a given type of multilayer structure.

$$
\left(\frac{d^{2}}{d r^{2}}+\frac{1}{r} \frac{d}{d r}+\left(\gamma_{i}^{2}-\frac{m^{2}}{r^{2}}\right)\right) y(r)=0
$$

where

$\gamma_{i}=\sqrt{n_{i}^{2} k_{0}^{2}-\beta^{2}}$, is the $i$ th layer mode wave number,

$y(r)$ is unknown function of the mode profile, 
$m$ is the mode angular symmetry number.

For a first order ordinary differential equation (ODE) of the type $y^{\prime}(t)=f(y, t)$, the numerical method starts from an initial poin $y_{0}$ and then each successive point $y_{1}, y_{2}, \ldots$ is computed. In other words, an algorithm should compute as precisely as possible the value of the function at the next step $y_{i+1}$ from the previous result $y_{i}$.

The equation (96) can be rewritten in the following form

$$
y^{\prime \prime}=f\left(y^{\prime}, y, r\right)=-\frac{1}{r} y^{\prime}-\left(\gamma_{i}^{2}-\frac{m^{2}}{r^{2}}\right) y
$$

The idea was to solve the equation (97) by substituting the values of the $\gamma_{i}=$ $\sqrt{n_{i}^{2} k_{0}^{2}-\beta^{2}}$ at each layer with it's own refractive index $n_{i}$.

As the first attempt of the numerical integration the Euler's method was used but then in order to increase precision the 4th order Runge-Kutta method chosen. Euler's method only uses first derivative information and has accumulated error of order $O(h)$, where $h$ the integration step size, while the Runge-Kutta method samples derivatives at several points in the interval and has accumulated error of order $O\left(h^{4}\right)$. The 4th order Runge-Kutta method also provides good trade-off between computational time and increased accuracy [32]. 


\subsection{Numerical integration method}

The 4th order Runge-Kutta method for a first order ODE $y^{\prime}=f(y, r)$ can be written in the form [32].

$$
\begin{aligned}
& y_{i+1}=y_{i}+\frac{h}{6}\left(k_{1}+2 k_{2}+2 k_{3}+k_{4}\right) \\
& r_{i+1}=r_{i}+h
\end{aligned}
$$

where

$$
\begin{aligned}
k_{1} & =f\left(r_{i}, y_{i}\right) \\
k_{2} & =f\left(r_{i}+\frac{h}{2}, y_{i}+\frac{h}{2} k_{1}\right) \\
k_{3} & =f\left(r_{i}+\frac{h}{2}, y_{i}+\frac{h}{2} k_{2}\right) \\
k_{4} & =f\left(r_{i}+h, y_{i}+h k_{3}\right)
\end{aligned}
$$

$h$ - is the step size,

$y$ - is the mode profile function.

The Runge-Kutta algorithm averages four slopes $k_{1}, k_{2}, k_{3}, k_{4}(99)$, with greater weight given to the slopes at the midpoint (Figure 13)

In order to solve equation (96) the above method should be modified for integration of a second order ODE $y^{\prime \prime}=f\left(y^{\prime}, y, r\right)$. Since higher order ordinary differential equations can be reduced to a system of first order ODE's, the modification is straightforward. 


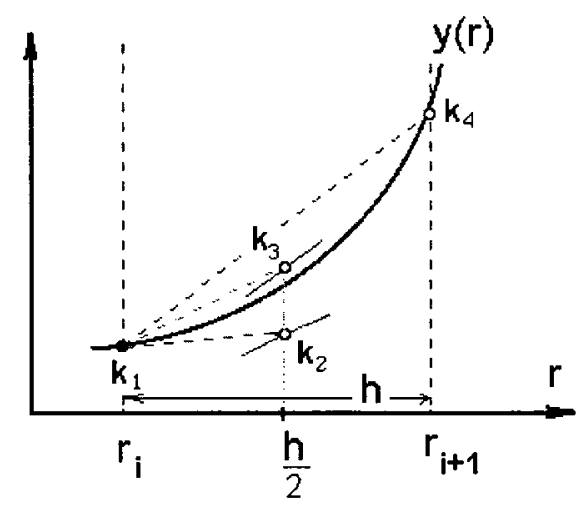

Figure 13: Schematic representation of the 4th order Runge-Kutta method

$$
\begin{aligned}
d y_{i+1} & =d y_{i}+\frac{1}{6}\left(k_{1}+2 k_{2}+2 k_{3}+k_{4}\right) \\
y_{i+1} & =y_{i}+\frac{1}{6}\left(d y_{i}+\left(k_{1}+k_{2}+k_{3}\right)\right) \\
r_{i+1} & =r_{i}+h
\end{aligned}
$$

where

$$
\begin{aligned}
& k_{1}=h f\left(r_{i}, y_{i}, d y_{i}\right) \\
& k_{2}=h f\left(r_{i}+\frac{h}{2}, y_{i}+d y_{i} \frac{h}{2}, d y_{i}+\frac{k_{1}}{2}\right) \\
& k_{3}=h f\left(r_{i}+\frac{h}{2}, y_{i}+d y_{i} \frac{h}{2}+k_{1} \frac{h}{2}, d y_{i}+\frac{k_{2}}{2}\right) \\
& k_{4}=h f\left(r_{i}+h, y_{i}+d y_{i} h+k_{2} \frac{h}{2}, d y_{i}+k_{3}\right)
\end{aligned}
$$

$h$ - is the step size,

$y$ - is the mode profile function,

$d y$ - is the derivative of $y$.

The latter numerical method (100) was used for integration of the equation (96). 


\subsection{Eigenvalues search algorithm}

As we can see from equation (96) it contains two unknown variables:

1) $m$ is the mode angular number, which defines modes type with respect to the angular symmetry,

2) $\gamma_{i}=\sqrt{n_{i}^{2} k_{0}^{2}-\beta^{2}}$ is defined by propagation constant $\beta=n_{e f f} k_{0}$ and the wavenumber $k_{0}$ of the incident light.

To account these variables we should replace our function $f\left(y^{\prime}, y, r\right)$ with the $f\left(y^{\prime}, y, r, m, n_{e f f}\right)$.

In order find eigenvalues for all propagating modes, the following algorithm was developed:

1) The mode angular symmetry number $m$ is chosen.

2) In order to find all eigenvalues $n_{\text {eff }}$ of the equation (96) the method of trial and error was used.

It is known that the eigenvalues of propagating cladding modes lay in the range of $n_{\text {clad }}<n_{\text {eff }}<n_{\text {core }}$. The idea was to scan that range of values with a small step, and decrease the step value when an eigenvalues was encountered.

The searching algorithm works as follows:

a) The eigenvalue is chosen from the range $\left[n_{\text {clad }} . n_{\text {core }}\right]$.

b) The equation (96) is solved for each of $y_{1}, y_{2}, \ldots$ points successively, for a chosen value of $n_{e f f}$. Usually after few hundred steps, the wave function $y(r)$ starts to diverge exponentially. This means that the the value of $n_{e f f}$ is incorrect.

c) Right before the function $y(r)$ blows up, its sign is stored, and the value of $n_{\text {eff }}$ is incremented by a small step $n_{\text {eff }}=n_{\text {eff }}+\Delta n$.

d) The process is repeated again for the new value $n_{\text {eff }}$ until the function $y(r)$ changes in sign right before diverging.

e) When the sign change is detected, the program will bisect the interval and will 
check each of the half intervals for a sign change. This procedure can be repeated $\mathrm{N}$ times to achieve a desired precision of eigenvalue.

The result of the above process is the function $y(r)$ goes through the whole area of interest without blowing up, and decays beyond the cladding region.

3) The mode symmetry $m$ can be changed and process of finding $n_{\text {eff }}$ can be repeated all over again for the new type of modes.

\subsection{Results and Discussion}

The numerical integration method and eigenvalues search algorithm described above were implemented in the program coded in Delphi 7.0 (see Appendix A for the program listing).

The graphical user interface of the program is presented in Figure 14. It consists of 1) a windows where structure parameters can be entered, 2) a window that displays computed eigenvalues for different mode types, 3) a window that displays a mode profile for a chosen eigenvalues $n_{e f f}$.

It can be seen that each eigenvalue is defined by two numbers and are placed in table. The table's column number is the $m$ number that defines the mode angular symmetry.

The presented program proved itself useful in the calculation of all the possible cladding modes in standard $125 \mu \mathrm{m}$ fibre. More than a hundreds modes were found on a standard Pentium 4 computer in less than ten minutes with eigenvalue precision up to nine orders of magnitude.

When a structure of interest is significantly bigger than the incident light wavelength, the numerical algorithm becomes ineffective due to the increasing number of steps. In this case methods with variable step size can be applied or analytical solution can be found for large cylindrical uniform regions. The program is based on 


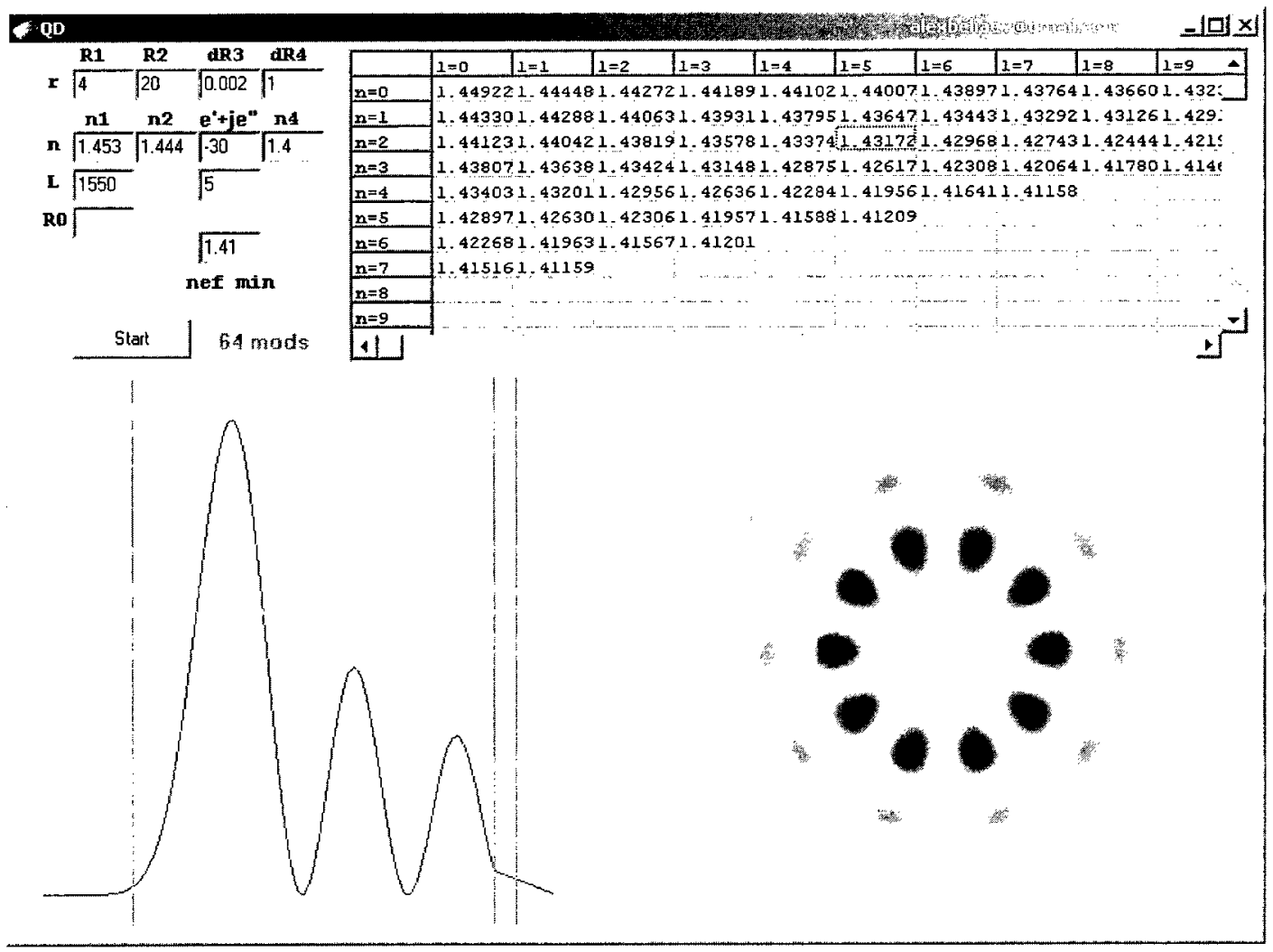

Figure 14: The graphical user interface of the program.

the linear polarization modes approximation.

Unfortunately this approach is not applicable for the structures where strong coupling between $\vec{E}$ and $\vec{H}$ fields exists on the layer boundaries. In the latter case the vectorial mode transfer matrix method should be used (see Chapter 3). 


\section{Chapter 5}

\section{TFBG sensor for nanoscale metal films}

\subsection{Introduction}

Fibre Bragg grating (FBG) sensors can be applied to a wide range of applications in pressure-strain, temperature and chemical process measurements. Optical FBG based sensors have several advantages over conventional electric sensors because they are inexpensive, small and immune to electromagnetic interference.

Present work shows how it is possible to extend FBG sensor application in chemistry for precise measurement of the fast kinetic process of metallic film deposition within nanometer precision. In this chapter we will describe developed TFBG chemical sensor and measurement technique used to monitor the fabrication of the nanoscale metal layer coatings.

Since mode field solution depends on the properties of all the layers making up the fibre, any changes to the layers will change the field shapes and hence the coupling coefficients, and finally the TFBG transmission spectrum. This was demonstrated elsewhere [33-35]

We will show that a TFBG can be used for a real-time in situ monitoring of thin metallic films deposition during a process of electroless plating. The technique presented in this work allows the precise measurement of metallic film thickness with 
precision of a few nanometers which opens the way for growing nanoscale metal coatings on fibres which is essential for obtaining optimal parameters for sensor, based on a surface plasmon resonance $[35,36]$.

The ultimate goal of this finding is to develop an inexpensive compact SPR sensing platform based on a standard optical telecom fibre coated with a thin metal film. The idea is to implement plasmon resonances to detect changes in environment and even separate molecular binding events. It known that such sensor is able to detect biomolecules at femto-molar concentration $[34,36]$ with the advantage of remote sensing in a small-size locations.

In general our method can either be used to fabricate TFBG-SPR sensors with excellent metal thickness accuracy, or as a process control to monitor precisely the batch fabrication of multiple coated fibres for any related application, since the electroless process can be used for large immersion baths and fibre numbers.

\subsection{Experiment Outline}

A tilted fibre Bragg grating (TFBG) sensor was used to monitor the growth of thin gold and silver metal films during a process of electroless plating.

To maximize the sensor response a linearly polarized light was introduced and measurements were taken at various different polarization angles. The linear polarization states at which the sensor showed the best performance were chosen.

The thickness of the metallic film coating was measured with the help of an atomic force microscope (AFM). Two methods were implemented to estimate the film thickness: 1) the method based on the direct observation of mechanically scratched film's surface areas, and 2) the method based on natural imperfections in the film coating.

The correlation between the sensor response and the film thickness was established. It was shown that the film thickness can be monitored at nanometer precision 
by looking at the optical response of the TFBG sensor.

\subsection{Metal film deposition}

The TFBG sensor was based on a standard single-mode optical telecoms fibres (Corning SMF-28). The phase mask technique was used to inscribe gratings in the fibre's hydrogen-loaded core. The mask was tilted to produce a 6 or 10 degrees tilt of the grating planes. The inscription was done with a $248 \mathrm{~nm}$ pulsed ultraviolet $\mathrm{KrF}$ excimer laser.

The metal film deposition was conducted through the process of electroless plating where gold nanoparticles were first attached to the fibre surface and used as catalysts for the metal deposition [37].

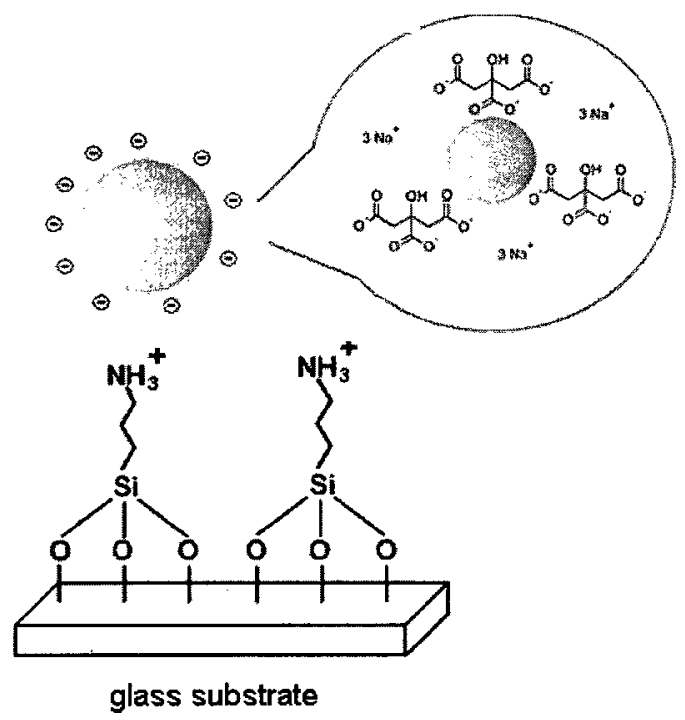

Figure 15: Schematic representation of AuNPs assisted electroless plating.

The chemical experiment was conducted as follows:

1) The gold nanoparticles (AuNPs) were synthesized by citrate reduction of chloroauric Acid $\left(\mathrm{H}\left[\mathrm{AuCl}_{4}\right]\right)$ in water $[38,39]$. The reducing agent sodium citrate 
$\left(\mathrm{Na}_{3} \mathrm{C}_{6} \mathrm{H}_{5} \mathrm{O}_{7}\right)$ reduces the gold ions to neutral gold atoms. AuNPs start to form when the colloidal solution becomes supersaturated. Citrate ions act as both a reducing agent, and a capping agent. The repulsion of the negatively charged citrate ions stabilize the colloid preventing the AuNPs from sticking together [39]. As the result of this process spherical gold nanoparticles in the range from 10 to $20 \mathrm{~nm}$ in diameter suspended in water were created.

2) The prepared Bragg grating fibres were immersed in concentrated sulfuric acid $\left(\mathrm{H}_{2} \mathrm{SO}_{4}\right)$ for 1 hour and then rinsed with deionized water and then with methanol.

In order to create a positively charged surface the fibre was then place in a $1 \%$ methanol solution of APTMS $\left((\mathrm{MeO})_{3} \mathrm{SiCH}_{2} \mathrm{CH}_{2} \mathrm{CH}_{2} \mathrm{NH}_{2}\right)$ for 15 minutes. The APTMS treatment replaces the hydroxyl groups $(\mathrm{OH})$ adsorbed on the glass (fibre) substrate $\left(\mathrm{SiO}_{2}\right)$ with APTMS molecules forming a siloxane bond between the $\mathrm{Si}$ on one end of the APTMS molecules and an oxygen atom on the $\mathrm{SiO}_{2}$ surface [40]. As a consequence, the amino group attached on the other end of the APTMS molecule is oriented away from the substrate. These amino groups on the APTMS molecules immobilise gold particles onto the substrate because of the affinity of the amino group to the gold $[40]$.

3) The fibre was removed from the $1 \%$ APTMS solution and rinsed a few times with methanol and then rinsed with deionized water. The fibre was then placed for 24 hours in a solution of citrate reduced gold nanoparticles with diameters ranging from 10 to $20 \mathrm{~nm}$.

4) The fibre was removed from the gold nanoparticles solution and rinsed with deionized water and immersed in the plating solution. The process of electroless plating was used to obtain a thin metallic film coating on the fibre surface (Figure 15). Electroless plating is a non-galvanic method of plating that involves several simultaneous reactions in an aqueous solution, which occur without the use of external 
electrical power [41]. The reaction is accomplished when hydrogen is released by a reducing agent and oxidized thus producing a negative charge on the surface of AuNPs. Nanoparticles attached to the fibre surface play the catalytic role during the process of electroless plating.

For the silver coating the plating solution consisted of $0.01 \mathrm{M}$ silver nitrate $\left(\mathrm{AgNO}_{3}\right), 8.96 \mathrm{M}$ ammonium nitrate $\left(\mathrm{NH}_{4} \mathrm{NO}_{3}\right), 2.24 \mathrm{M}$ acetic acid $\left(\mathrm{CH}_{3} \mathrm{COOH}\right)$ and $0.4 \mathrm{M}$ hydrazine hydrate $\left(\mathrm{H}_{2} \mathrm{NNH}_{2} . \mathrm{H}_{2} \mathrm{O}\right)$ at a volumetric ratio of $1: 1: 1: 2$, respectively.

For the gold plating solutions of $0.1 \%$ hydrogen tetrachloroaurate ( $\mathrm{H} \mathrm{AuCl}_{4} .3 \mathrm{H}_{2} \mathrm{O}$ ) and $0.4 \mathrm{M}$ hydroxylamine hydrochloride $\left(\mathrm{NH}_{2} \mathrm{OH} \mathrm{HCl}\right)$ in a $1: 1$ volumetric ratio were used.

The process described resulted in the metalilic coating of the fibre. Figures 16(a), 16(b) presents the AFM scans done before and after process of metal film deposition.

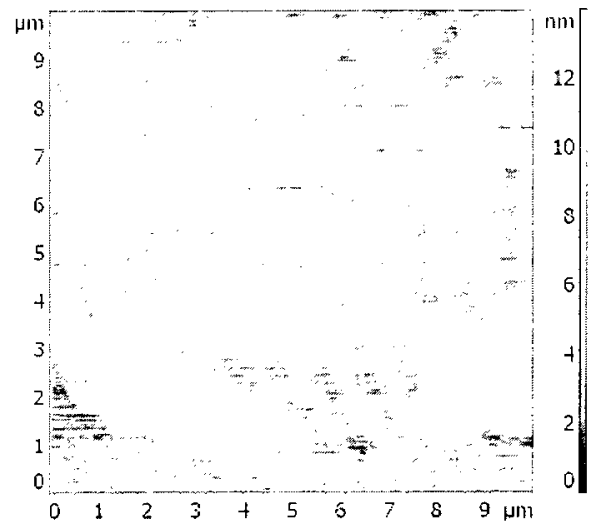

(a)

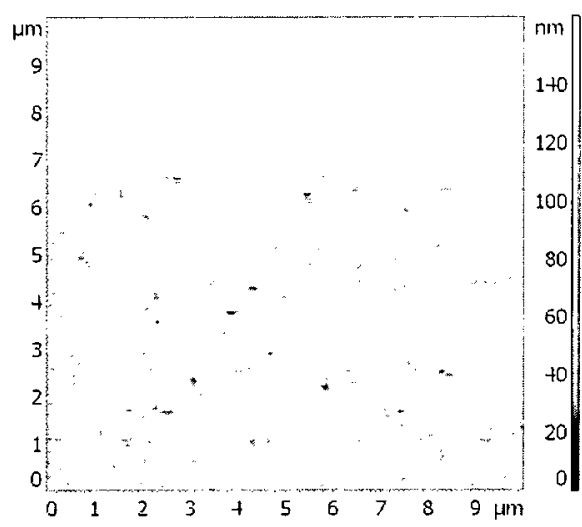

(b)

Figure 16: AFM scan of the fibre coated with (a) AuNPs with 24h of deposition time, (b) the gold film with 32 minutes of deposition. 


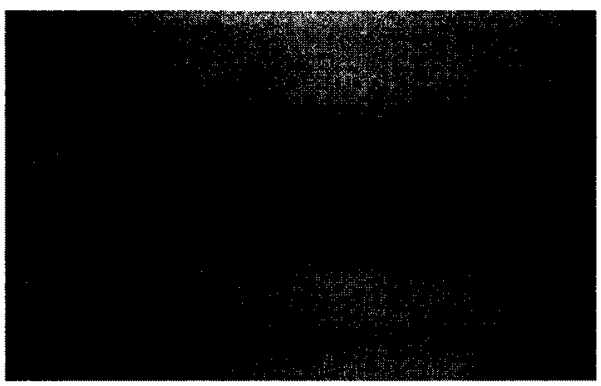

Figure 17: Image of the fibre coated with the gold film taken from an optical microscope.

\subsection{Optical system and TFBG response}

The experimental setup for real-time monitoring of the process of formation of thin gold and silver metal films is shown schematically in Figure 22.

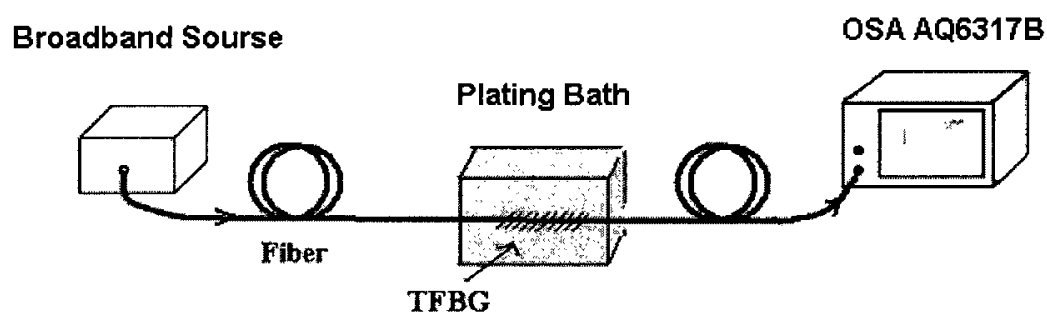

Figure 18: Schematic diagram of the optical system.

The light from a broadband source was coupled to the optical fibre connected to the TFBG sensor. Transmission spectra were measured by an optical spectrum analyzer AQ6317B. The optical spectrum analyzer AQ6317B provides measurements with high spectral resolution but unfortunately measurements are done relatively slowly (usually one scan per minute can be done). The whole process of gold nanoparticles and metal film deposition was measured and automated. Data were gathered by a PC connected to the optical spectrum analyzer through a LabView interface. The collected data were analyzed with help of MatLab software.

Figure 19 (a) shows a single spectrum taken by the optical spectrum analyzer. 
Spectra were taken continuously and saved on a PC hard drive for further analysis. Figure 19 (b) shows a $3 \mathrm{D}$ representation of the spectra, ploted with a MatLab program.

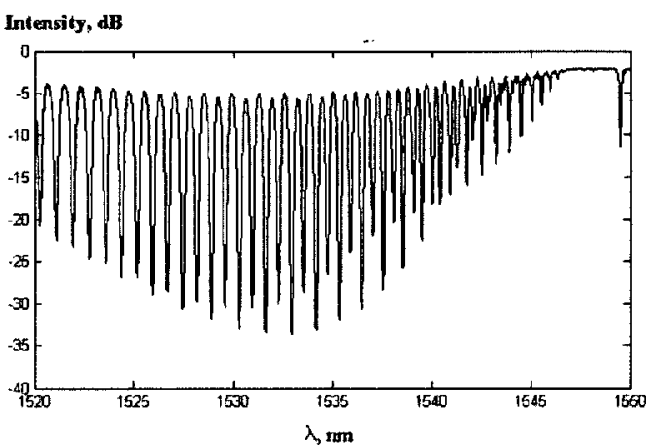

a)

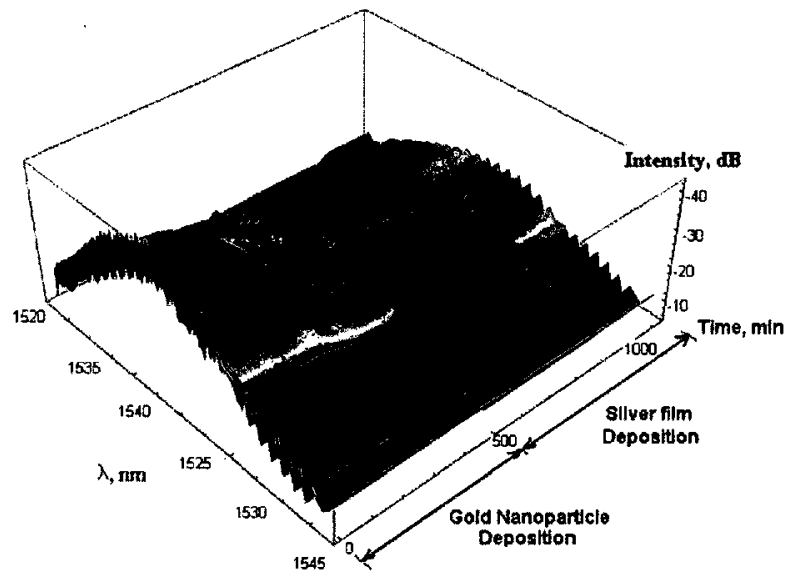

b)

Figure 19: (a) Typical transmission spectrum of a weakly tilted FBG, (b) a 3D representation of a set of the TFBG spectra taken at different deposition time.

The data representation can be improved if the intensity of each spectrum's resonance is tracked separately regardless of its wavelength shift. It can be seen from Figure 19 (a) that the TFBG spectrum consists of two different groups of resonances. Figure 20 shows spectrum envelope for one of these group, where each spectrum's resonance was tracked separately.

Figure 20 shows distinct changes in the spectrum envelope during the different stages of the deposition process. The evolution of the spectra envelope identifies many different features. The biggest change is related to the change of plating solution from AuNPs to the metal plating, is the result of refractive index change. Due to the high resolution of the optical spectrum analyzer AQ6317B it was possible to have a close look at individual resonances during the change of plating solution (figure 21). The fine structure of each single resonance can be observed. Figure 21 clearly shows that during the deposition process different families of cladding modes are influenced by 


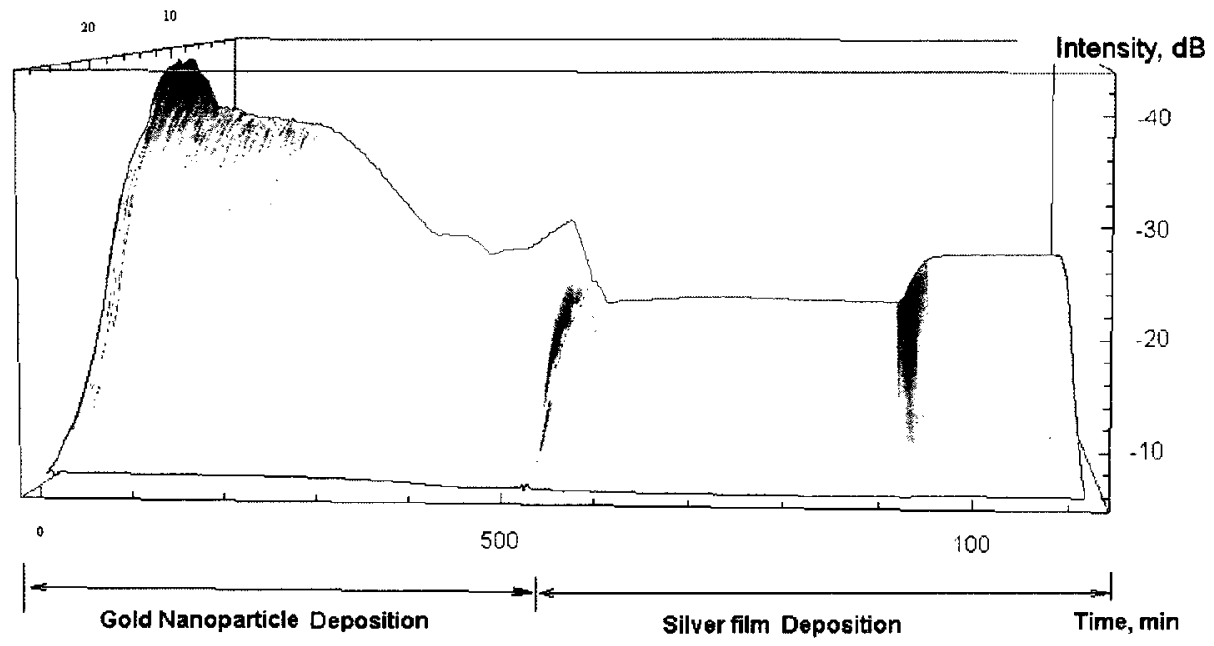

Figure 20: Evolution of the spectrum envelope during the whole process of deposition.

the environment differently.
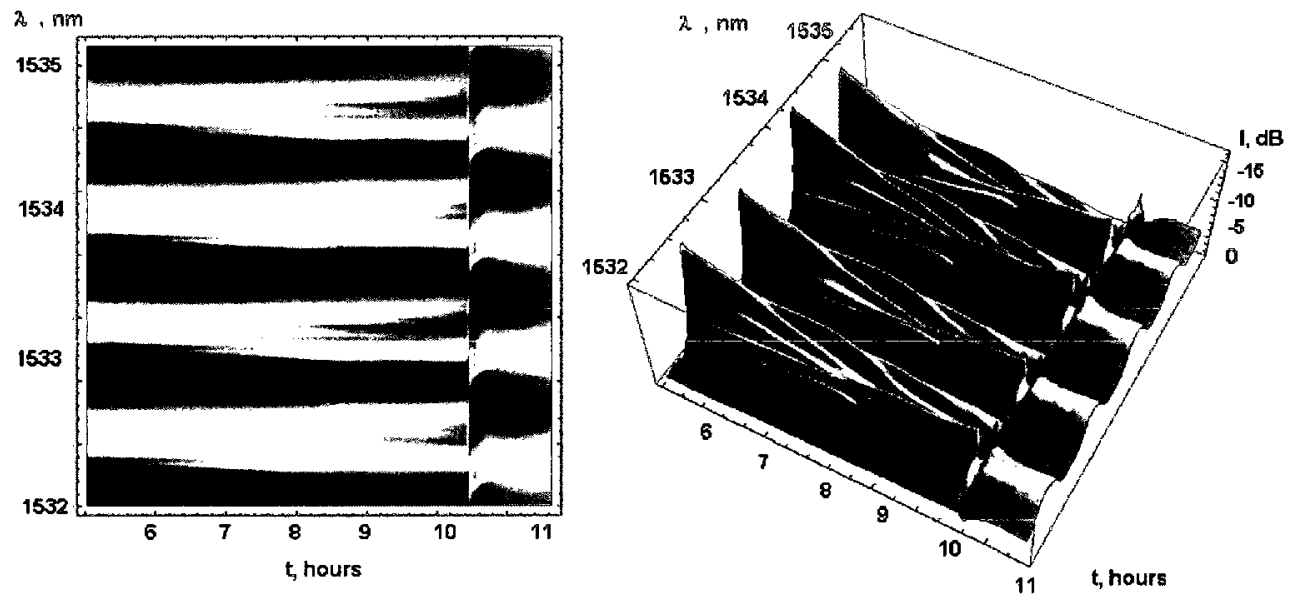

Figure 21: Density and 3D plot of individual resonances.

After getting the whole picture the research was focused on the sub-process of metal deposition in the first 30 minutes of metal plating. The goal was to estimate the metal film thickness growth during the deposition process.

Unfortunately the first few experiments showed no significant changes in the 
TFBG transmission spectruma except the first few minutes. To improve the sensor performance the idea of using polarized light was introduced. Changes in the core mode polarization of the TFBG sensor causes excitation of different mode groups in the cladding. It was expected to see the difference in sensor response when different groups of cladding modes were excited, because of metal deposition.

A new optical setup was introduced, as shown in Figure 22.

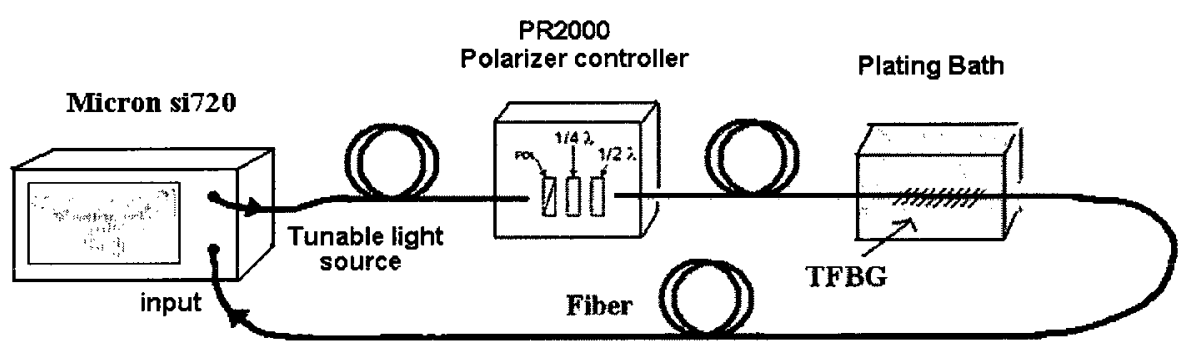

Figure 22: Schematic diagram of the optical system with a polarizer controller added.

In order to control the light state of polarization a PR2000 automated polarization rotator was introduced (Figure 22). In this system (Figure 22), light from a fibre goes through a polarization controller that sets the light states of polarization before it reaches the TFBG sensor. The required state of polarization was produced by aligning fast axes of $1 / 4$ wave and $1 / 2$ wave retarders with a micro-stepped motor. The PR2000 polarizer controller is capable of rotating light polarization through all polarization states in the range ot 1530 to $1560 \mathrm{~nm}$.

The optical spectrum analyzer AQ6317B was replaced by si720 Micron Optical Sensing Interrogator, based on a high-speed Fabry-Perot tunable filters and fast photo detecting system, capable to measure 5 spectra per second, although with lower spectral resolution in comparison with initial AQ6317B. The high speed of spectra measurement allowed to track the chemical process kinetics.

The polarization controller was set to continuously scan all linearly polarized states, performing full rotation every 10 seconds. Meanwhile, the optical spectrum 
analyzer (Micron si720) was capable of acquiring 5 spectra per second with reasonable resolution, which gives $5 * 10=50$ measurements per full polarization rotation. Measurements were continuously taken in the range of 1530 to $1560 \mathrm{~nm}$ during 30 min. As the result 180 spectra were accrued for each of 50 different polarization states during the metal deposition process (Figure 23).
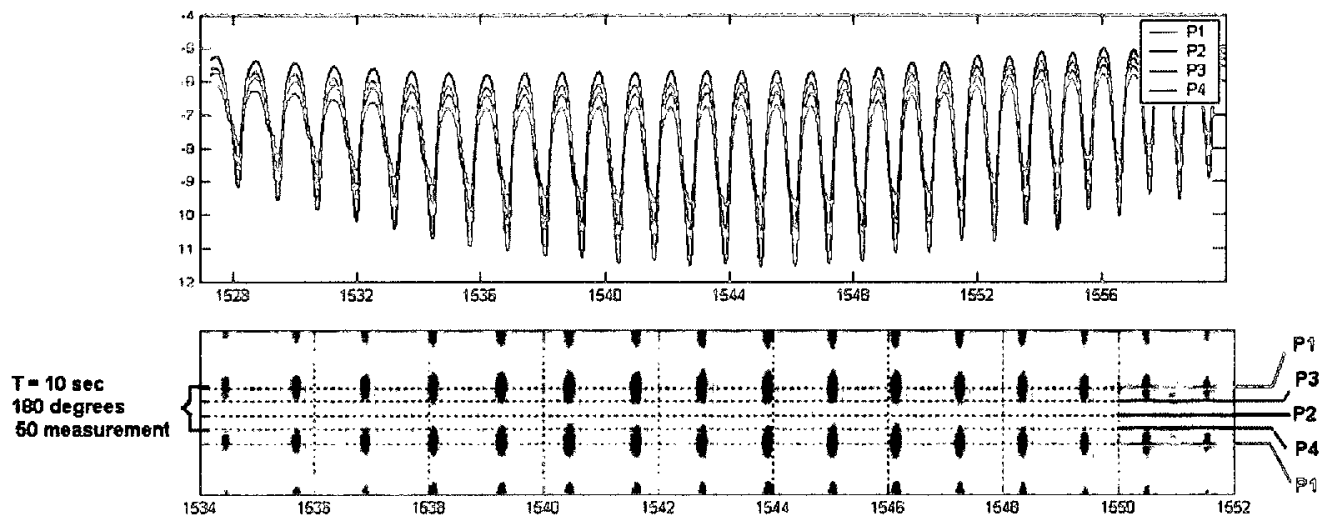

Figure 23: The density plot representation of spectra acquired for the first $30 \mathrm{~min}$ of the gold film deposition

This system acquires a lot of data. Prior to the experiment we did not know what would be happening and how the TFBG response would vary. The following section indicates a multi-step data analysis process with increasingly finer resolution designed to identify the metal plating process.

Extracting data for each of 50 measured states makes it is possible to track spectrum evolution for each of 50 states of polarization. As shown in Figure 23, four different states of polarization: P1, P2, P3, P4, corresponding to the axes defined by the grating tilt plane, were chosen for further analysis. These confirm the fact that the growth of the metal layer influences modes differently, depending on the polarization of their EM field at the cladding boundary.

Figure 24 shows spectrum evolution of P3 state (as defined in Figure 23).

By tracking each resonance in the spectrum separately for each of 50 states of 


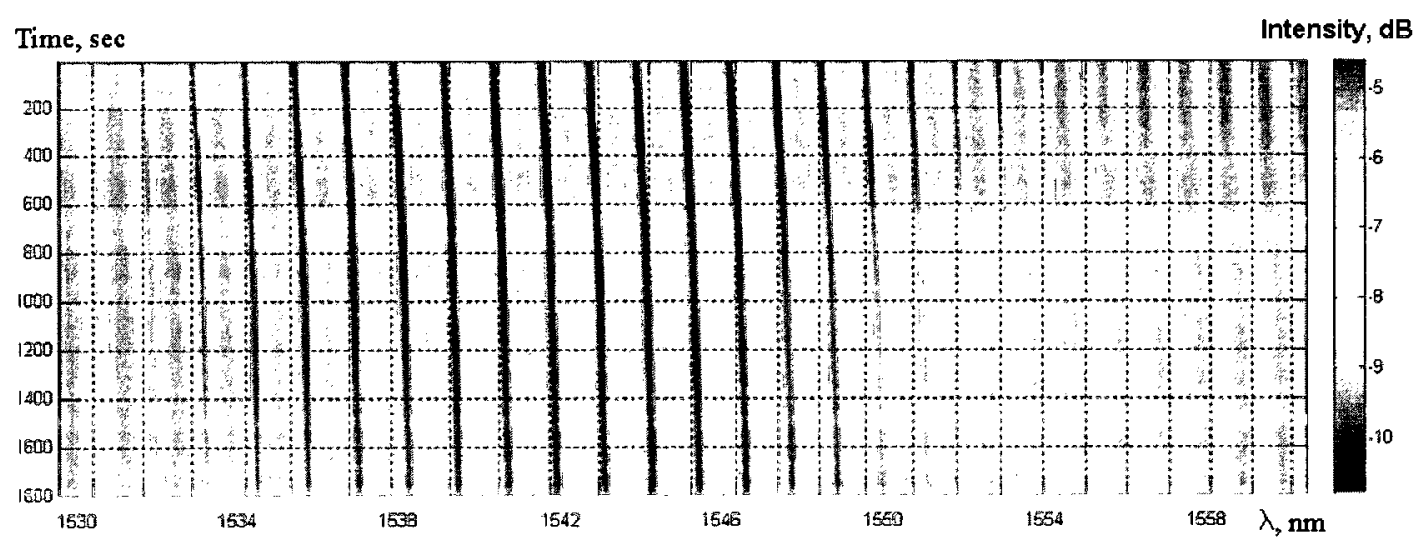

Figure 24: Density plot of the spectrum evolution for the P3 polarization state $(\lambda=1530.1560 \mathrm{~nm}, t=1 . .1800 \mathrm{sec}$.

polarization, the full description of the TFBG sensor response was ascertained. Figure 25 shows the amplitude evolution of one of such resonances for input light with different polarization states. This particular resonance at $1531 \mathrm{~nm}$ was chosen as it has an almost linear amplitude-time dependence for P2 polarization state, which is used in further analysis.

It is clearly seen from Figure 25 that the polarization state of the incident core's mode has a significant influence on the TFBG sensor response. The most valuable information is provided by the P1 and P2 states of linear polarizations, while the results from the P3 and P4 states are more immune to the deposition process. Moreover, the TFBG sensor response to the P1 and P2 states has an opposite sign, which explains the cancellation effect for unpolarized light, when no sensor response was ascertained during the film deposition process.

The next step was to measure the metallic film thickness in order to corelate the optical response of the TFBG sensor (Figure 25) with the actual physical results of deposition. The expected film thickness was in the range of 5 to $100 \mathrm{~nm}$. In order to measure such a thin coating an atomic force microscope (AFM) was used. 


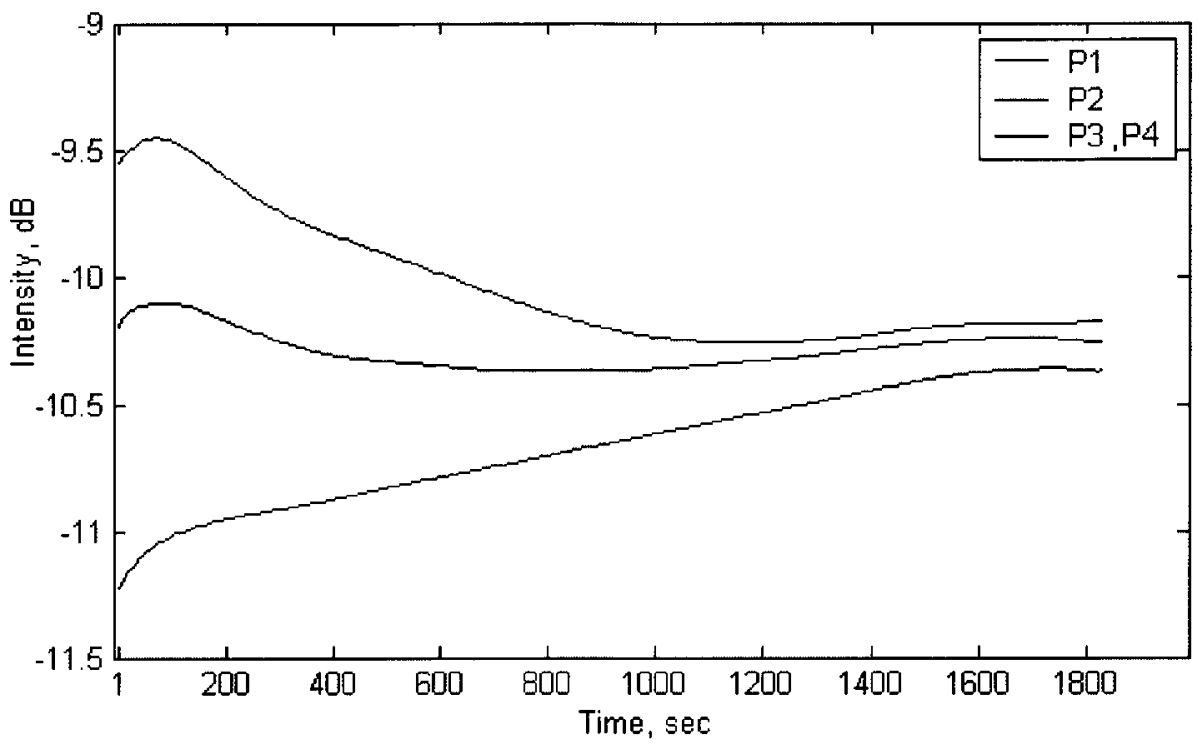

Figure 25: Evolution of individual resonance near $1531 \mathrm{~nm}$ for P1,P2,P3 polarization states

\subsection{Metal film thickness estimation}

\subsubsection{Principles of AFM operation}

To control the coating process and estimate the film thickness the atomic force microscope (AFM) was implemented with the capability to provide resolution on the order of a nanometer. AFM has several advantages as follows: it provides a true three-dimensional surface profile, and samples do not require any special treatments or coatings. The process works perfectly well in ambient air.

Essentially during AFM measurements the information is gathered by sensing the surface with a mechanical probe.

The AFM Figure 26 consists of a reflective cantilever with a sharp tip attached to its underside. When the tip is brought close to the sample surface, forces between the tip and the sample lead to a deflection of the cantilever. Depending on the situation, forces that are measured in AFM include short-range force associated with this chemical interaction and long-range forces arising from van der Waals and 
electrostatic interactions between tip and surface. A diode laser is focused onto the back of a reflective cantilever. When the tip scans across the surface, moving up and down due to roughness on the surface, the tip will interact with the surface causing the cantilever to deflect according to the interaction. The cantilever deflection is measured by a laser spot reflected from the the cantilever into an array of photodiodes. The difference in the light intensity between different photodiodes in the array is transformed into voltage and acquired by a control circuit. In most cases a control feedback mechanism is employed to adjust the tip-to-sample distance to maintain a constant force between the tip and the sample or stay at constant height above the sample. The tip is mounted on a vertical piezo z-scanner, through which constant force is maintained, while the sample is scanned in $\mathrm{x}$ and $\mathrm{y}$ directions with use of other piezo blocks. Essentially from this z-movement of the sensor and $\mathrm{x}, \mathrm{y}$ movement of the sample the resulting three dimensional topographical maps of the surface of the sample is obtained.

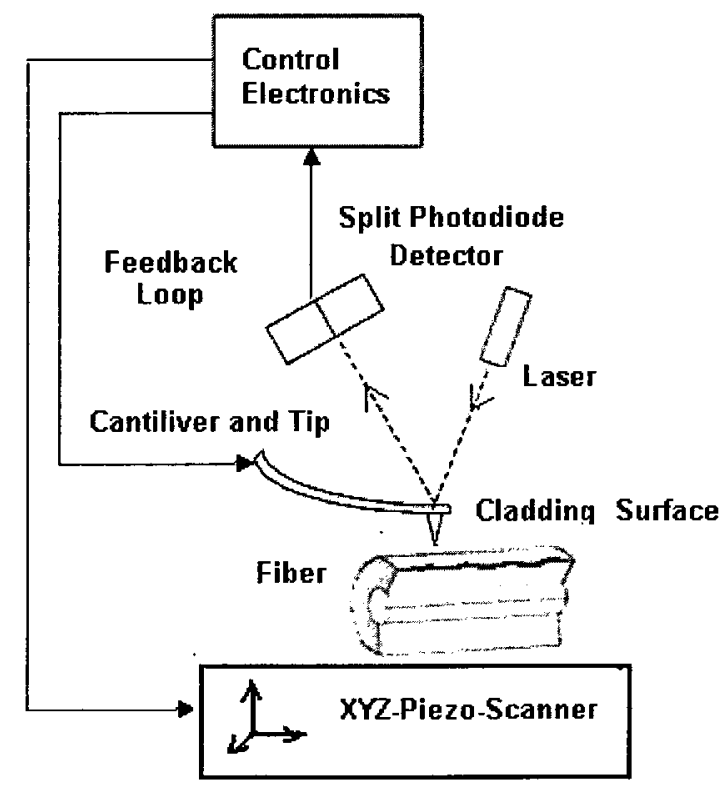

Figure 26: The tip is attached to a cantilever, and is $x, y$-scanned over a surface. The cantilever deflection due to tip-surface interactions is monitored by a photodiode. 
An AFM can be operated in a number of modes, depending on the application. In general, possible modes are divided into a contact mode and a variety of dynamic modes where the cantilever is vibrated.

In this work the contact and tapping modes were implemented. The contact mode had showed better performance, but sample and tip were damaged a few times.

\section{Contact Mode}

In this mode the tip scans the sample in close contact with the surface. The deflection of the cantilever is sensed and compared in a DC feedback amplifier to some desired value of deflection. If the measured deflection is different from the desired value the feedback amplifier applies a voltage to the piezo element to change the cantilever's height and thus restores the desired value of deflection. Thus the force between the tip and the surface is kept constant during scanning by maintaining a constant deflection.

Because the measurement of a static signal is more exposed to noise and drift, lowstiffness cantilevers are used to increase the deflection signal. However, low stiffness cantilevers can be easily polled to the surface by attractive forces. Thus static mode AFM is almost always done in contact where the overall force is repulsive.

In practice, it appears that the contact AFM mode is strongly influenced by formation of electrostatic and surface tension forces from the adsorbed gas layer pull the scanning tip toward the sample surface. These frictional forces are destructive and can damage the sample and the cantilever probe as well as distort the resulting data. Therefore, contact mode imaging is heavily influenced by frictional and adhesive forces compared to non-contact or tapping mode.

An attempt to avoid these problem is the non-contact mode. 


\section{Non-contact Mode}

In this mode, the tip of the cantilever does not contact the sample surface. It is implemented in situations where tip contact might alter the sample in subtle ways. The tip is usually from 1 to $10 \mathrm{~nm}$ above the sample surface. The cantilever is given an oscillation by a small piezoelectric element mounted in the AFM tip holder at a frequency slightly different from the resonance frequency with an oscillation amplitude of a few nanometers. The van der Waals forces act to decrease the resonance frequency of the cantilever. AC detection methods are used to detect the change in frequency, amplitude or phase of the oscillating cantilever in response to small perturbations in force between the tip and the sample. A feedback loop system maintains a constant oscillation amplitude or frequency by adjusting the average distance from tip to the sample.

Non-contact mode AFM does not suffer from tip or sample degradation effects, which makes this mode preferable for measuring soft samples. Unfortunately, the attractive forces from the sample are substantially weaker than the forces used by contact mode. Non-contact scans usually have lower resolution than in contact mode and can also be influenced by a contaminant layer which can interfere with oscillation.

\section{Tapping Mode}

To detect short-range forces the probe tip should be close enough to the sample and at the same time it should be prevented from sticking to the surface. The idea of the tapping mode is to alternately place the tip in contact with the surface to provide high resolution and then lift the tip off the surface to avoid dragging the tip across the surface. The tapping mode was developed as a method to achieve high resolution without introducing destructive frictional forces.

In Tapping Mode, the cantilever is driven to oscillate near its resonance frequency 
similar to non-contact mode but with higher amplitude typically greater than $20 \mathrm{~nm}$ at a frequency of $50-500 \mathrm{KHz}$. The oscillating tip is then moved toward the surface until it begins to lightly touch, or tap the surface. During scanning, the vertically oscillating tip alternately contacts the surface and lifts off. As the oscillating cantilever begins to intermittently contact the surface, the cantilever oscillation is reduced due to energy loss caused by the tip contacting the surface. The reduction in amplitude is used to measure the surface features.

Tapping mode prevents the tip from sticking to the surface and reduces the damage during scanning in comparison with contact mode. Unlike contact mode, where the tip contacts the surface, it has sufficient oscillation amplitude to overcome the tipsample adhesion forces, therefore material is not pulled sideways by the tip. 


\subsubsection{AFM Measurement technique}

\section{AFM Setup}

To obtain the value of the metal film thickness with the AFM it is highly important to choose optimal values for certain parameters that control AFM operation. The following two parameters have major impacts:

1) The seating point - tip height over the sample surface.

2) The feedback (FB) - parameter which controls the feedback loop and affecting the resolution of the image.

Both the seating point and the feedback parameters should be tuned. Usually one starts from setting the tip height and then picking the optimal value of the feedback loop parameter for a given height. If the FB is too large a parasitic oscillation might occur as the result of the tip overreaction. If the FB signal is too small the tip would not be able to follow the rapid changes in the sample's surface height variation and the image becomes too general with loss of fine details. If the results are not satisfactory the seating point should be changed.

3) In the taping mode there are extra two parameters: a) the voltage applied to the cantilever, and b) signal amplification coefficient. It is possible to pick one of the cantilever's resonances, and then control the cantilever's oscillation amplitude by adjusting the applied voltage and the coefficient of signal amplification.

These parameters had crucial significance for measurements as the goal was to detect film thickness by looking at small imperfections in coating, with rapid changes in height. When the tip passes over a bump in the surface, the cantilever has less room to oscillate and the amplitude of oscillation decreases. Alternately, when the tip passes over a pitch the cantilever has more room to oscillate. If the control system is not set up properly, oscillations can reach a free space value and then information related to the film structure would be lost. After performing control scans and choosing the 


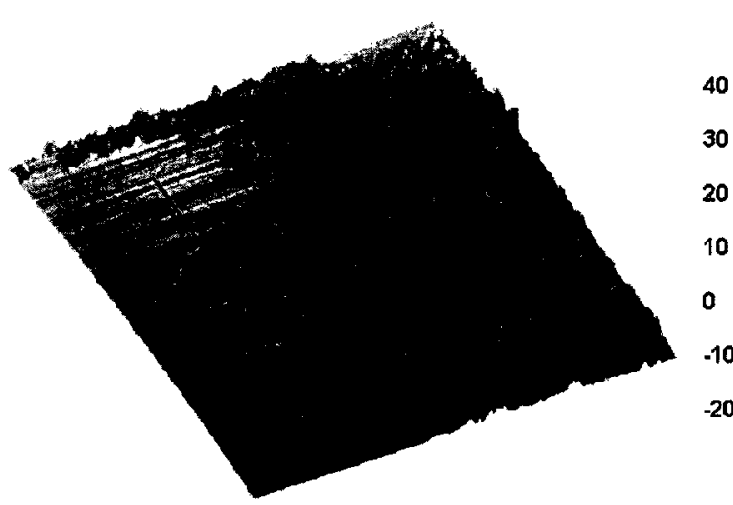

(a)

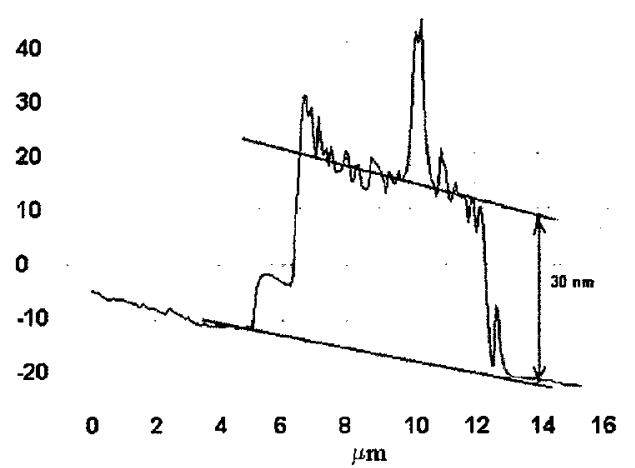

(b)

Figure 27: (a) The scratched metallic film,(b) The film height estimation.

value for the parameters it was possible to perform fine calibrations with the AFM software to effectively reduce nonlinear distortions.

\section{Film thickness measurement techniques}

There were two major goals: to measure the metallic film thickness and to estimate the coating quality. To achieve them two different methods were applied:

1) The film surface was mechanically scratched (Figure 27(a)) and then the AFM measurements were conducted on the scratch boundary. It was possible to perform measurements at different scratches in different film spots and to obtain the information regarding the film thickness.

The major drawback of this technique was that it is hard to perform precise scratches without affecting the film structure. The second problem was that it difficult to position the scan probe over the scratch boundary.

2) In the attempt to solve the above problems we have introduced a new method of measuring film thickness based on the detection of natural defects in a coating.

The process of chemical deposition of a thin metallic silver or gold film starts with the fibre surface activation by gold nanoparticles. The first stage of the film 


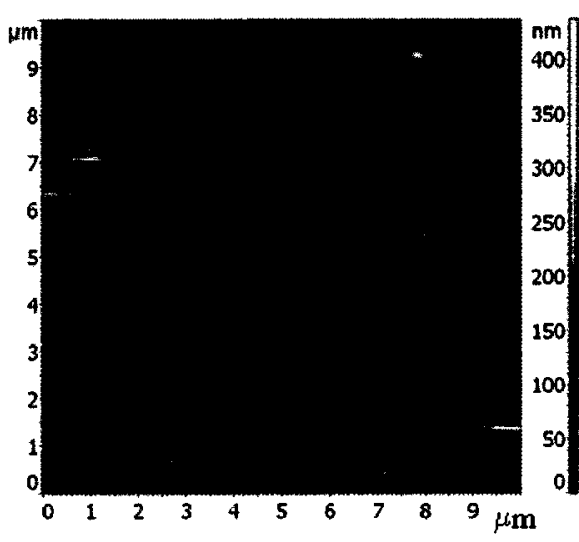

(a)

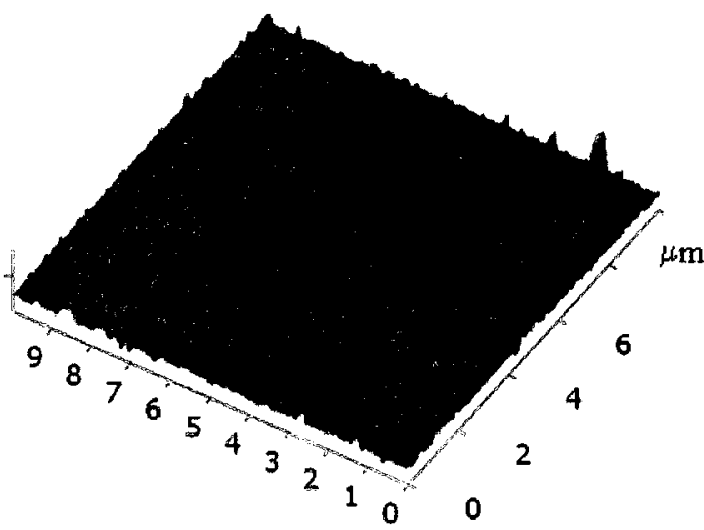

(b)

Figure 28: (a) Flattened AFM Image, 2D density plot, (b) Flattened AFM Image, 3D plot

growth leads to the formation of small islands which grow in size as time progresses. At the final stage of deposition these islands start to overlap producing a more or less uniform coating. However there always several small uncovered spots. Thus it was possible to estimate the gold film thickness by analyzing unscratched AFM scans and estimate the film roughness. The software provided with the AFM provides data in both $3 \mathrm{D}$ image and matrix formats. The matrix contains numbers representing height of each scanned point in the xy plane. It was possible to calculate the number of matrix elements within a certain range of values and plot the histogram graph.

The fibre's surface curvature need to be considered first because it causes the sample height variation significantly greater than the gold film roughness. One way to flatten the graph is to perform second order curve subtraction, where parameters of the second order curve are chosen in the way to provide as flat image as possible. This easily can be done as the height variation related to the fibre curvature is a slow varying function in compression with the function associated to the film roughness. Figure 28(b) present the result of curvature subtraction.

The next step was the surface roughness and thickness estimation. For each AFM 


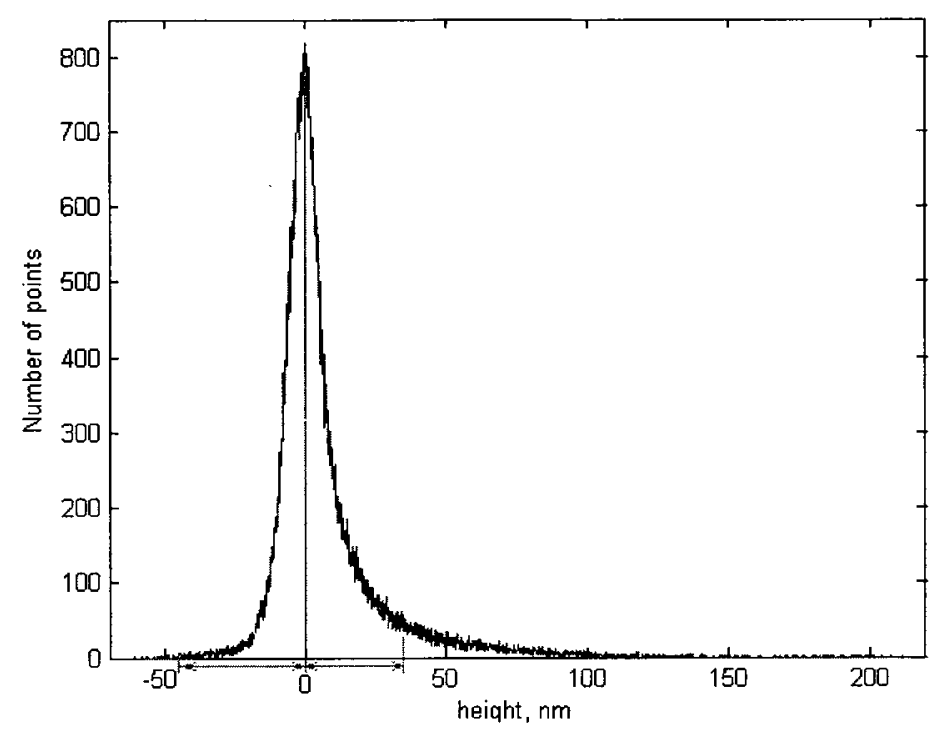

Figure 29: Histogram representation of a film surface.

scan a histogram was plotted. Figure 29 represents the frequency distribution for different points.

Analyzing the histogram in Figure 29, it is clear to see that it has a distinct peak, which represents the most frequent value of the film height occurring on the film. The tails on the left and right hand sides represent deviation from the film's most frequent height value. Thus, for a good uniform coating the peak is narrow and sharp. The left most side of the histogram is representative of badly-coated areas on the film surface, which either have thinnest coating or no coating whatsoever.

The film thickness was ascertained by measuring the distance between the histogram's peak and the left end side of the histogram tail (Figure 29). The results of film thickness estimation were in good correlation with the first method described above.

The graph in Figure 30 represent results of roughness estimation for the gold films with a varying deposition times.

To remove the high frequency noise and obtain more useful data, a low pass filter 


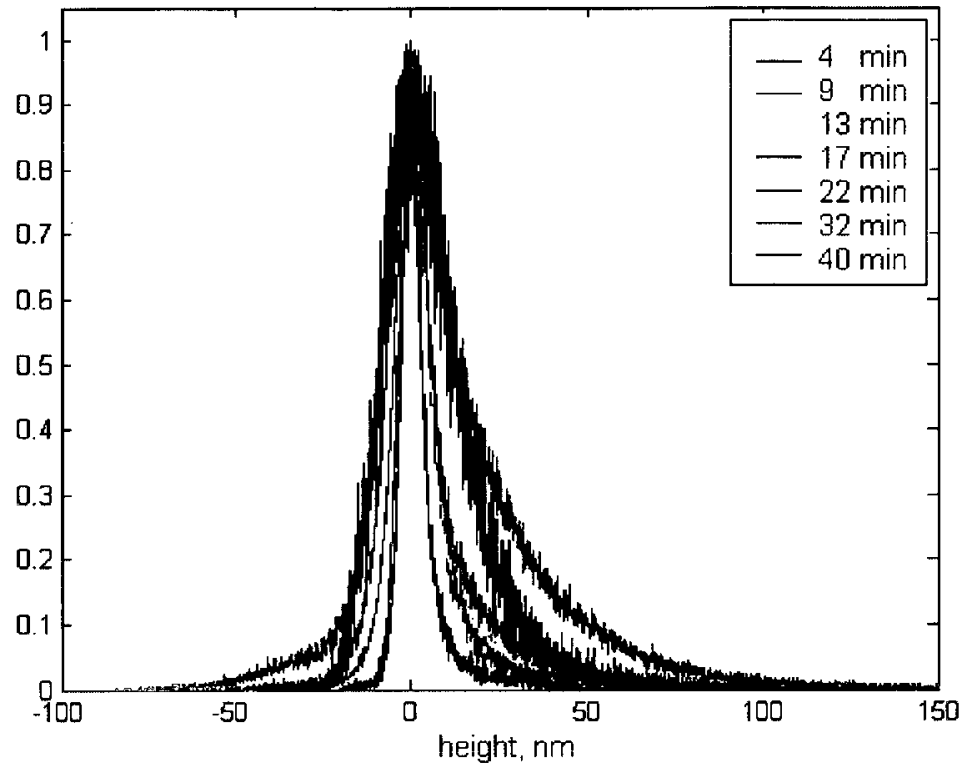

Figure 30: Histogram of AFM scans of the gold film with different exposure time.

was applied in the frequency domain (Figure 31).

By close and careful inspection of graphs in Figure 32 the gold film thickness was plotted as the function of deposition time (Figure 33). The linear and cubic interpolations were implemented to display the trend of the process. 


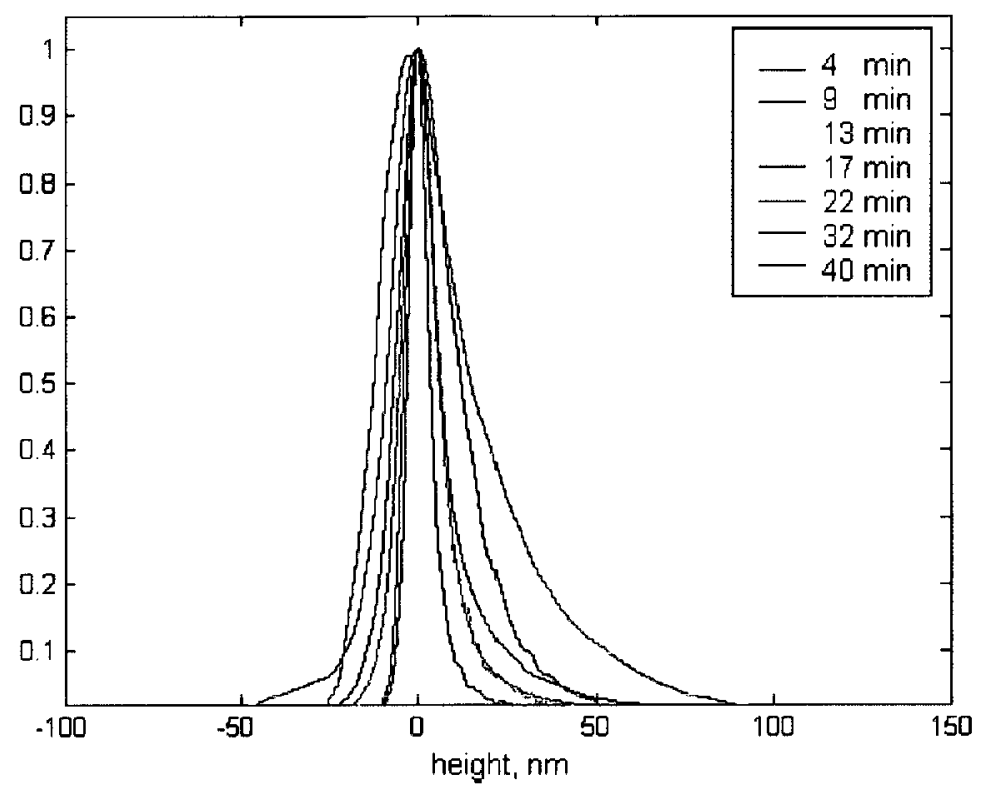

Figure 31: Histograms filtered with a low pass "brick" filter.

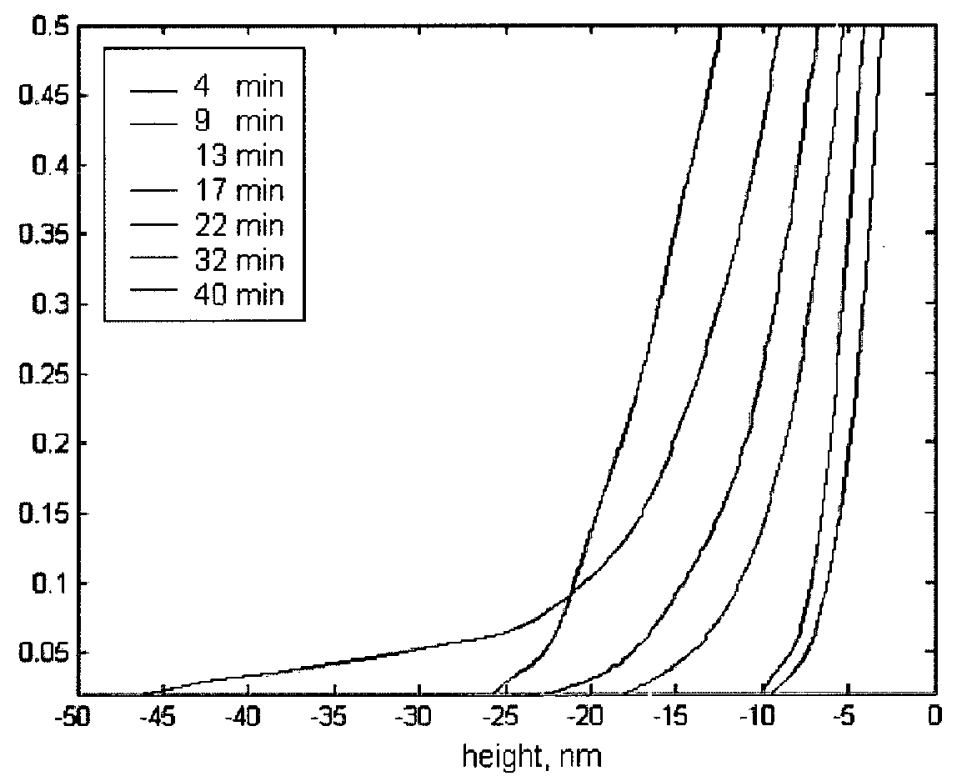

Figure 32: Zoom of Figure 31 (Intersections with the "height" axis gives the value of the gold film thickness measured from the film surface to the fibre substrate). 


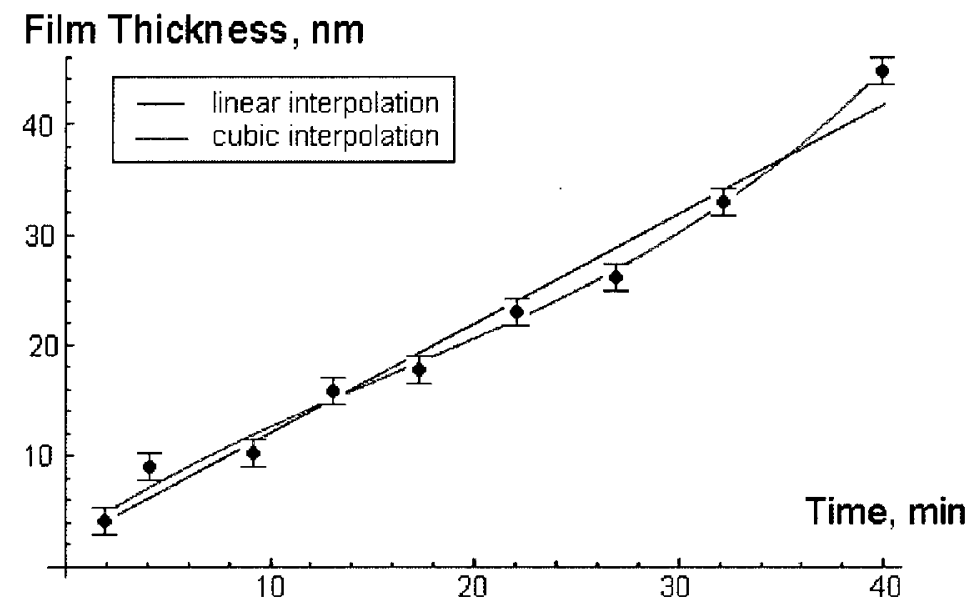

Figure 33: The gold film thickness vs deposition time, with linear and cubic interpolation.

\subsection{Results and Discussion}

Metal plating results in significant changes to the transmission spectrum of the TFBG as a function of time. Using the TFBG sensor optical response (Figure 25) and AFM measurements of the gold film thickness (Figure 33) it was possible to calibrate the sensor platform (Figure 34).

As seen in Figure 34 the experimental results can be interpolated with a second order polynomial. The standard deviation (102) is on the order of nanometer. The interpolation curve in Figure 34 can be used for predicting the film thickens in realtime measurements. It is clearly seen that changes in metal film thickness as small as few nanometers are easily detectable.

$$
s=\sqrt{\frac{1}{N} \sum_{i=1}^{N}\left(x_{i}-f\left(y_{i}\right)\right)^{2}}=1.0890, n m
$$

The polarized spectral timeliness contain a tremendous amount of data (Figure 23). When a polarized light is launched into the fibre it causes excitation of different families of cladding modes, affected differently by the sensing environment. 

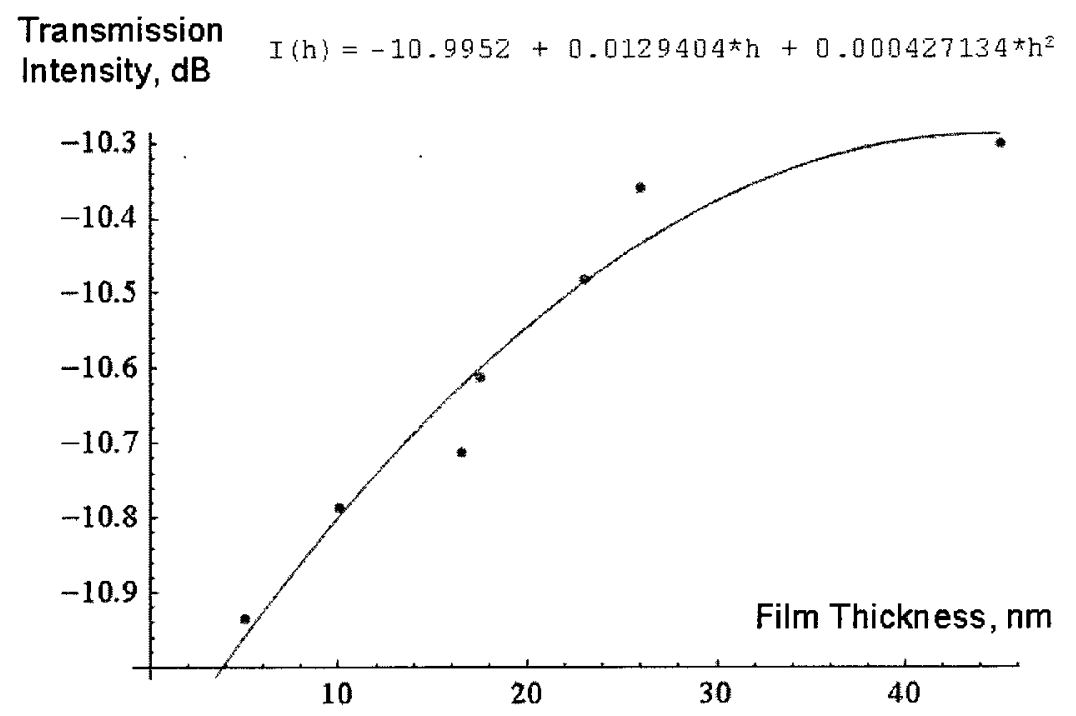

Figure 34: Gold film thickness vs intensity for one particular cladding mode resonance at $1531 \mathrm{~nm}$. The line represents the second order polynomial interpolation.

However, only few states of polarization provid valuable information. Moreover, it was showed that optical response of the sensor can be opposite for different polarization states, which leads to cancellation of the sensor response when unpolarized light is used.

Overall, the sensor showed excellent performance. It was capable of detecting fabrication of thin metallic film with nanometer precision and high time resolution. The proposed platform can also be applied for chemical process kinetics detection. With the help of the si720 Micron Optical Sensing Interrogator measurements can be taken with frequency of $5 \mathrm{~Hz}$, whereas a si920 sensing interrogator can take measurements at microsecond time resolution. 


\section{Chapter 6}

\section{Conclusions and Future Work}

We have shown that chemical deposition of thin metallic films can be monitored with the help of TFBG cladding mode resonances. The presented sensing platform was capable of detecting changes in metal thickness as small as few nanometers, via real-time monitoring of the metal plating process.

The proposed method can be used to produce TFBG-SPR sensors with excellent metal thickness accuracy, where the thickness of the metal coating is precisely tuned to obtain the plasmon resonance in the working range of the TFBG sensor. This method can also be used in any related application such as fabrication of multiple coated fibres.

A major advancement was made when polarized light was introduced, and sensor response at different states of polarization was ascertained. The proposed method was based on the single polarization state tracking ( $P_{1}$ or $P_{2}$ in Figure 25), at which the sensor had the larger response. This technique can be improved if the difference between $P_{1}$ or $P_{2}$ (Figure 25) states is taken into account. Although, in a real-life applications it would require a more complicated setup, capable of providing separate detection of the sensor response at two different polarization states $P_{1}$ or $P_{2}$.

It is known that SPR is very sensitive to light polarization $[34,35]$, and this was exactly the case in the presented experiment. Moreover, at certain stage of 
the deposition process, a sharp peak in the spectrum was observed for a particular polarization state $P_{2}$ (Figure 35), suggesting the energy coupling from the cladding modes to the surface plasmon resonance wave in the film cladding. As described elsewhere $[34,35]$, coupling to the SPR wave can be expected only when the metallic film has a particular thickness, which was the case in the presented results.

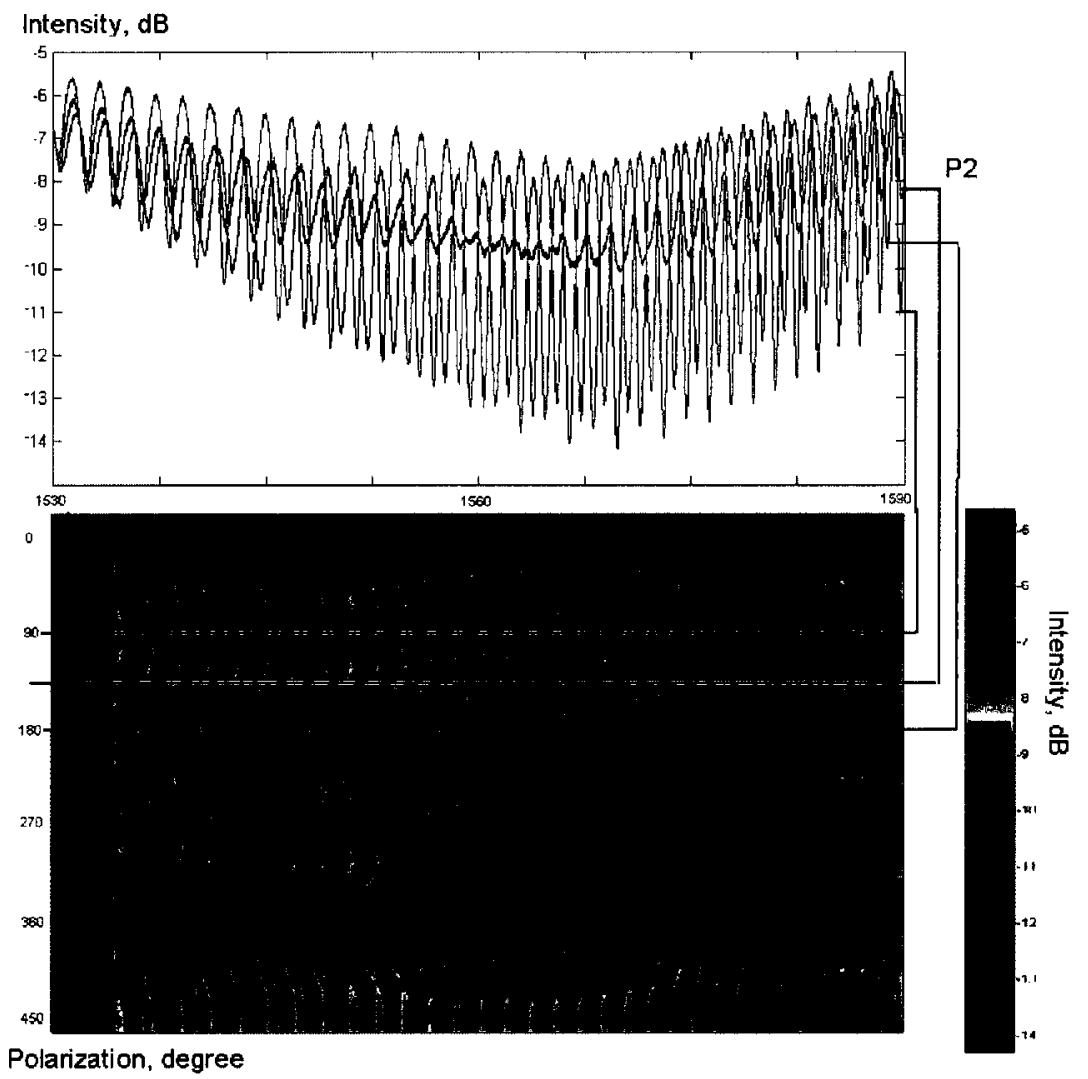

Figure 35: Possible SPR detection at P2 polarization state (black line) after 40 min of gold film deposition

Overall, the polarized spectral timelines contain a tremendous amount of data. Much work still remains to fully correlate all the features we see with the physical processes occurring during plating.

The numerical method developed in Chapter 4 provides a fast and accurate solution to the fibre modes problem for a multilayer step-index structures with a slowly 
varying refractive index function.

Unfortunately, this is not the case when a metal layer is taken into account. Strong coupling between fields $\vec{E}$ and $\vec{H}$ on the boundary makes it impossible to decouple the system of Maxwell's equations. In this case the vectorial mode transfer matrix method, derived in Chapter 3, should be used for further simulation. 


\section{List of References}

[1] K. O. Hill, Y. Frujii, D. C. Johnson, and B. S. Kawasaky, "Photosensitive in optical fiber waveguides: Applications to reflection filter fabrication," Applied Physics Letters, vol. 32, pp. 647-649, 1978.

[2] D. Lam and B. Garside, "Characterization of single mode optical fibers," Applied Optics, vol. 20, pp. 440 445, 1981.

[3] G. Meltz, W. W. Morey, and W. H. Glam, "Formation of bragg grating in optical fibers by a transverse holographic method," Optics Letters, vol. 14, pp. 823-825, 1989.

[4] K. O. Hill, B. Malo, F. Bilodeau, D. C. Johnson, and J. Albert, "Bragg gratings fabricated in monomode photosensitive optical fibers by uv exposure through a phase mask," Applied Physics Letters, vol. 62, pp. 1035-1037, 1993.

[5] P. J. Lemaire, R. M. Atkins, V. Mizrahi, K. L. Walker, K. S. Kranz, and W. A. Reed, "High pressure h2 loading as a technique for achieving ultrahigh uv photosensitivity and thermal sensitivity in geo2 doped optical fibers," Electronics Letters, vol. 29, pp. 1191-1193, 1993.

[6] H. Hosono, Y. Abe, L. Donald, A.Robert, K. Muta, and H. Kawazoe, "Nature and origin of the 5-ev band in sio2:geo2 glasses," Physical Review Letters B, vol. 46, pp. 11445-11451, 1992.

[7] D. P. Hand and P. S. J. Russell, "Photoinduced refractive-index changes in germanosilicate fibers," Optics Letters, vol. 15, pp. 102-104, 1990.

[8] P. D. Townsend and J. C. Kelly, Colour Centres and Imperfections in Insulators and Semiconductors. John Wiley and Sons, 1973.

[9] R. M. Atkins, V. Mizrahi, and T. Erdogan, "248 nm induced vacuum uv spectral changes in optical fibre preform cores: support for a colour centre model of photosensitivity," Electronics Letters, vol. 29, pp. 385-387, 1993. 
[10] R. Kashyap, Fiber Bragg Gratings. Academic Press, 1999.

[11] J. Nishii, K. Fukumi, H. Yamanaka, K. Kawamura, H. Hosono, and H. Kawazoe, "Photochemical reactions in geo2-sio2 glasses induced by ultraviolet irradiation: Comparison between hg lamp and excimer laser," Physical Review Letters B, vol. 52, pp. 1661-1665, 1995.

[12] B. G. Potter and J. K. Simmons-Potter, "Photosensitive point defects in optical glasses: Science and applications," Nuclear Instruments and Methods in Physics Research Section B, vol. 166, pp. 771-781, 2000.

[13] A. Lanin, O. Butov, and K. Golant, "Response of in-fiber bragg gratings to hydrogen loading and subsequent heat treatment in h2 ambience," Applied Optics, vol. 45, pp. 5800-5807, 2006.

[14] J. Pierce, "Coupling of modes of propagation," Journal of Applied Physics, vol. 25, pp. 179-183, 1956.

[15] A. Snyder, "Coupled-mode theory for optical fibers," Journal of The Optical Society of America, vol. 62, pp. 1267-1277, 1972.

[16] A. Yariv, "Coupled-mode theory for guided-wave optics," Journal of Quantum Electronics, vol. QE-9, pp. 919-933, 1973.

[17] T. Erdogan, "Fiber grating spectra," Journal of Lightwave Technology, vol. 15, pp. 1277-1294, 1997.

[18] F. Ouellette, "Dispersion cancellation using linearly chirped bragg grating filters in optical waveguides," Optics Letters, vol. 12, pp. 847-847, 1987.

[19] M. Fermann, K. Sugden, and I. Bennion, "High-power soliton fiber laser based on pulse width control with chirped fiber bragg gratings," Optics Letters, vol. 20, pp. 172-174, 1995.

[20] N. Matuschek, F. Kartner, and U. Keller, "Exact coupled-mode theories for multilayer interference coatings with arbitrary strong index modulations," Journal of Quantum Electronics, vol. 33, pp. 295-302, 1997.

[21] T. Erdogan, "Cladding-mode resonances in short- and long-period fiber grating filters," Journal of The Optical Society of America, vol. 14, pp. 1760-1773, 1997.

[22] K. S. Lee and T. Erdogan, "Fiber mode coupling in transmissive and reflective tilted fiber gratings," Applied Optics, vol. 39, pp. 1394-1404, 2000. 
[23] T. Guo, A. Ivanov, C. Chen, and J. Albert, "Temperature-independent tilted fiber grating vibration sensor based on cladding-core recoupling," Optics Letters, vol. 39, pp. 1004-1006, 2008.

[24] K. Kawano and T. Kitoh, Introduction to Optical Waveguide Analysis: Solving Maxwell's Equations and the Schrodinger Equation. John Wiley and Sons, 2001.

[25] K. Iizuka, Elements of Photonics. For Fiber and Integrated Optics. John Wiley and Sons, 2001.

[26] D. Kqjfez and P. Guillon, Dielectric Resonators. Noble Publishing Corporation, 1998.

[27] A. Snyder and J. Love, Optical Waveguide Theory. Chapman and Hall, 1983.

[28] K. Okamoto, Fundamentals of Optical Waveguides. Elsevier, 2006.

[29] C. Yeh and F. Shimabukuro, The Essence of Dielectric Waveguides. Springer, 2008.

[30] C.-L. Chen, Foundations for Guided-wave Optics. John Wiley and Sons, 2007.

[31] F. Bouttout, F. Lethimonnier, and D. L. Bihan, "Full-wave analysis of single cylindrical striplines and microstriplines with multilayer dielectrics," International Journal for Numerical Methods in Engineering, vol. 68, pp. 809-835, 2006.

[32] K. Atkinson, An Introduction to Numerical Analysis. John Wiley and Sons, 1989.

[33] C. F. Chan, C. Chen, A. Jafari, A. Laronche, D. J. Thomson, and J. Albert, "Optical fiber refractometer using narrowband cladding-mode resonance shifts," Applied Optics, vol. 46, pp. 1142-1149, 2007.

[34] L. Shao, Y. Shevchenko, and J. Albert, "Intrinsic temperature sensitivity of tilted fiber bragg grating based surface plasmon resonance sensors," Optics Express, vol. 18, pp. 11464-11471, 2010.

[35] Y. Shevchenko and J. Albert, "Plasmon resonances in gold-coated tilted fiber bragg gratings," Optics Letters, vol. 32, pp. 211-213, 2007.

[36] P. C. Lee and D. Meisel, "Adsorption and surface-enhanced raman of dyes on silver and gold sols," The Journal of Physical Chemistry, vol. 86, pp. 3391-3395, 1982. 
[37] L. Porter, J. Choi, A. Ribbe, and J. Buriak, "Controlled electroless deposition of noble metal nanoparticle films on germanium surfaces," Nano Letters, vol. 10, pp. 1067-1071, 2002.

[38] J. Turkevich, P. Stevenson, and J. Hillier, "A study of the nucleation and growth processes in the synthesis of colloidal gold," Discuss. Faraday. Soc., vol. 11, pp. 55-75, 1951.

[39] M. Daniel and D. Astruc, "Gold nanoparticles: Assembly, supramolecular chemistry, quantum-size-related properties, and applications toward biology, catalysis, and nanotechnology," Chemical Review, vol. 11, pp. 293-346, 2004.

[40] T. Sato, D. Brownb, and B. Johnsonb, "Nucleation and growth of nano-gold colloidal lattices," Chemistry Communications, vol. 11, pp. 1007-1008, 1997.

[41] M. Paunovic and M. Schlesinger, Fundamentals of electrochemical deposition. John Wiley and Sons, 2006. 


\section{Appendix A}

\section{Listing of program for analysing multilayer cylindrical structures}

uses

Windows, Messages, SysUtils, Variants, Classes, Graphics, Controls, Forms, Dialogs, StdCtrls, ExtCtrls, Menus, Grids, Buttons, Math;

type

TForm $1=\operatorname{class}($ TForm $)$

StringGrid1: TStringGrid; test: TButton; Er1: TEdit; Label1: TLabel; Er2: TEdit;

Er3: TEdit; Er4: TEdit; Label4: TLabel; En1: TEdit; En2: TEdit; En3: TEdit;

En4: TEdit; Label2: TLabel; Label5: TLabel; Label6: TLabel; Ejn: TEdit; Label7:

TLabel; Elamda: TEdit; Er0: TEdit; Label9: TLabel; Label11: TLabel; Label12:

TLabel; Label13: TLabel; Enmin: TEdit; Label3: TLabel; Label8: TLabel; Label14:

TLabel; Label10: TLabel;

function $\mathrm{kr}(\mathrm{r}, \mathrm{E}: \mathrm{real}$; l:integer):real;

function f(r:real; y, dy: extended; E:real; l:integer ):extended;

procedure Start;

procedure testClick(Sender: TObject);

procedure DU(l:integer; E:real); 
procedure DUsign(l: integer; E: real; var s : integer);

procedure Efind;

procedure Cnorm(L,n: integer);

procedure Init(L:integer; E:real);

procedure FormPaint(Sender: TObject);

procedure PlotPsi(L,n: integer);

procedure PlotPsi2(L,n: integer);

procedure PlotClear;

procedure StringGrid1SelectCell(Sender: TObject; ACol, ARow: Integer;

var CanSelect: Boolean);

end;

var

Form1: TForm1;

dr,ri,rmax,rmin: real; yi,dyi :extended;

$\mathrm{R} 1, \mathrm{R} 2, \mathrm{R} 3, \mathrm{R} 4, \mathrm{n} 1, \mathrm{n} 2, \mathrm{n} 3, \mathrm{n} 4, \mathrm{nj}, \mathrm{kk} 0$,Emin,Emax,dE,lamda :single;

Cn: Array [1..50] of real;

ENL,CNL,YNL,RNLmax,RNLmin: Array [0..50, 1..50] of real;

EnMax: Array [1..50] of integer;

rimax,rimin, yimin: real;

mods,Lmax: integer;

Const

$\mathrm{y} 0=1 \mathrm{E}-2$

implementation

$/ /++++++++++++++++++++++++++++++++++++$

$/ /+++$ Code 
procedure TForm1.Start;

begin

lamda:=StrToFloat(Elamda.Text)/1000;

$\mathrm{kk0}:=\operatorname{Power}\left(2^{*}\right.$ pi/lamda,2);

$\mathrm{R} 1:=$ StrToFloat(Er1.Text);

R2:=StrToFloat(Er2.Text);

R3:=StrToFloat(Er3.Text)+R2;

R4:=StrToFloat(Er4.Text)+R3;

$\mathrm{dr}:=0.01$

n1:=StrToFloat(En1.Text);

$\mathrm{n} 2:=$ StrToFloat(En2.Text);

n3:=StrToFloat(En3.Text);

n4:=StrToFloat(En4.Text);

$\mathrm{nj}:=\operatorname{Str}$ ToFloat$($ Ejn.Text);

Emin:=StrToFloat(Enmin.Text);

Emax: $=\mathrm{n} 1$;

$\mathrm{dE}:=0.003$

end;

$/ /++++++++++++++++++++++++++++++++++++$

$/ /+++$ Functions

Function TForm1.kr(r,E:real;1:integer):real;

var er: real;

begin

if $r>R 4$ then er: $=1 / /$ air

else if $r>R 3$ then er: $=n 4^{*} n 4 / /$ cover 
else if $r>R 2$ then er: $=\mathrm{n} 3 / /$ metal

else if $\mathrm{r}>\mathrm{R} 1$ then er: $=\mathrm{n} 2^{*} \mathrm{n} 2 / /$ clading

else er: $=\mathrm{n} 1{ }^{*} \mathrm{n} 1 ; / /$ core

$\mathrm{kr}:=\mathrm{kk} 0^{*}\left(\mathrm{er}-\mathrm{E}^{*} \mathrm{E}\right) \mathrm{l}^{*} \mathrm{l} /\left(\mathrm{r}^{*} \mathrm{r}\right)$

end;

Function TForm1.f(r:real; y, dy: extended; E:real; l:integer ):extended;

begin

$\mathrm{f}:=-\mathrm{dy} / \mathrm{r}-\mathrm{kr}(\mathrm{r}, \mathrm{E}, \mathrm{l}){ }^{*} \mathrm{y}$;

end;

$/ / 1++++++++++++++++++++++++++++++++++++$

$/ /+++$ ODE equation solution

// Runge-Kutta method

procedure TForm1.DU(L:integer; E:real);

var $\mathrm{t}, \mathrm{k} 1, \mathrm{k} 2, \mathrm{k} 3, \mathrm{k} 4$ : extended;

begin

$\mathrm{t}:=\mathrm{Abs}(\mathrm{kr}(\mathrm{ri}, \mathrm{E}, \mathrm{L}))$;

if $\mathrm{t}>20$ then $\mathrm{dr}:=0.00001$

else if $\mathrm{t}>10$ then $\mathrm{dr}:=0.0001$

else if $\mathrm{t}>5$ then $\mathrm{dr}:=0.001$

else $d r:=0.01$

$\mathrm{k} 1:=\mathrm{dr} * \mathrm{f}(\mathrm{ri}, \mathrm{yi}, \mathrm{dyi}, \mathrm{E}, \mathrm{L})$;

$\mathrm{k} 2:=\operatorname{dr} * \mathrm{f}(\mathrm{ri}+\mathrm{dr} / 2$, yi+dyi*dr/2, dyi $+\mathrm{k} 1 / 2, E, \mathrm{~L}) ;$

$\mathrm{k} 3:=\mathrm{dr}{ }^{*} \mathrm{f}\left(\mathrm{ri}+\mathrm{dr} / 2, \mathrm{yi}+\mathrm{dyi}{ }^{*} \mathrm{dr} / 2+\mathrm{k} 1 * \mathrm{dr} / 2, \mathrm{dyi}+\mathrm{k} 2 / 2, \mathrm{E}, \mathrm{L}\right) ;$

$\mathrm{k} 4:=\mathrm{dr}{ }^{*} \mathrm{f}\left(\mathrm{ri}+\mathrm{dr}, \mathrm{yi}+\mathrm{dyi}{ }^{*} \mathrm{dr}+\mathrm{k} 2^{*} \mathrm{dr} / 2, \mathrm{dyi}+\mathrm{k} 3, \mathrm{E}, \mathrm{L}\right) ;$ 
dyi: $=$ dyi $+\left(\mathrm{k} 1+2^{*} \mathrm{k} 2+2^{*} \mathrm{k} 3+\mathrm{k} 4\right) / 6$

yi: $=y i+d^{*}(d y i+(k 1+k 2+k 3) / 6)$;

$\mathrm{ri}:=\mathrm{ri}+\mathrm{dr}$;

end;

$/ 1++++++++++++++++++++++++++++++++++++$

$/ /+++$ Initial declaration

procedure TForm1.Init(L:integer;E:real);

begin

$\operatorname{rmax}:=\mathrm{R} 4+2$

$\mathrm{ri}:=0.00001$;

if $\mathrm{L}=0$ then begin $\mathrm{yi}:=\mathrm{y} 0$; dyi: $=0$; end;

if $L>0$ then

begin

repeat

$\mathrm{ri}:=\mathrm{ri}+\mathrm{dr}$

until $\operatorname{Abs}(\mathrm{kr}(\mathrm{ri}, \mathrm{E}, \mathrm{L}))<30$;

$\mathrm{yi}:=\mathrm{y0}$;

dyi:=Sqrt $(\operatorname{Abs}(\operatorname{kr}(\mathrm{ri}, \mathrm{E}, \mathrm{L})))^{*} \mathrm{y} 0$;

end;

rimin: $=$ ri

end;

$/ /++++++++++++++++++++++++++++++++++++$ $/ /+++$ Sign 
procedure TForm1.DUsign(L:integer; E: real; var s : integer);

var $\mathrm{t} 1, \mathrm{t} 2$ : real;

begin

$\mathrm{t} 1:=0 ; \mathrm{t} 2:=0$;

$\operatorname{Init}(\mathrm{L}, \mathrm{E})$;

repeat

$\mathrm{DU}(\mathrm{L}, \mathrm{E})$;

$\mathrm{t} 2:=\mathrm{Abs}(\mathrm{yi})$;

if $\mathrm{t} 2>1 \mathrm{E} 5$ then break;

if $\mathrm{t} 1>\mathrm{t} 2$ then rimax: $=\mathrm{ri}$;

$\mathrm{t} 1:=\mathrm{t} 2$

until ri $>$ rmax;

if $y i<0$ then $s:=1$ else $s:=-1$;

end;

procedure TForm1.Cnorm(L,n:integer);

var Int,YM,t: real;

begin

$\operatorname{Init}(\mathrm{L}, \mathrm{ENL}[\mathrm{L}, \mathrm{n}])$;

Int: $=0$;

$\mathrm{YM}:=0$;

repeat

DU(L,ENL[L,n]);

$\mathrm{t}:=\mathrm{yi} * \mathrm{yi}$

Int:=Int $+t^{*} \mathrm{dr}$;

if $\mathrm{t}>\mathrm{YM}$ then $\mathrm{YM}:=\mathrm{t}$;

until ri $>$ RNLmax $[\mathrm{L}, \mathrm{n}]$; 
$\operatorname{Cn}[\mathrm{n}]:=1 /$ Int;

YNL[L,n]:=YM;

end;

$/ /++++++++++++++++++++++++++++++++++++$

$/ /+++$ Eigenvalues Ei and Norm coefficients $\mathrm{Ci}$

procedure TForm1.Efind;

var

$\mathrm{L}, \mathrm{i}, \mathrm{j}, \mathrm{s} 0, \mathrm{~s} 1, \mathrm{~s} 2$ : integer;

E, dEd: real;

begin

$\operatorname{mods}:=0$

for $\mathrm{i}:=1$ to 50 do for $\mathrm{j}:=0$ to 50 do $\operatorname{ENL}[\mathrm{j}, \mathrm{i}]:=0$;

for $\mathrm{i}:=0$ to 50 do $\operatorname{EnMax}[\mathrm{i}]:=0$;

for $\mathrm{L}:=0$ to 50 do begin

$\mathrm{j}:=1$;

$\mathrm{E}:=\mathrm{Emax}$

$\mathrm{DU} \operatorname{sign}(\mathrm{L}, \mathrm{E}, \mathrm{SO})$;

repeat

$\mathrm{E}:=\mathrm{E}-\mathrm{dE}$;

$\mathrm{dEd}:=-\mathrm{dE}$;

$\operatorname{DUsign}(\mathrm{L}, \mathrm{E}, \mathrm{s} 1)$;

if $\mathrm{s} 1<>\mathrm{s} 0$ then

begin

for $\mathrm{i}:=1$ to $18 \mathrm{do}$

begin 
$\mathrm{dEd}:=\mathrm{dEd} / 2$;

$\mathrm{E}:=\mathrm{E}-\mathrm{dEd}$

$\operatorname{DUsign}(\mathrm{L}, \mathrm{E}, \mathrm{s} 2)$;

if $\mathrm{s} 1<>\mathrm{s} 2$ then begin $\mathrm{s} 0:=\mathrm{s} 2 ; \mathrm{E}:=\mathrm{E}+\mathrm{dEd}$; end;

nd;

$\operatorname{ENL}[\mathrm{L}, \mathrm{j}]:=\mathrm{E} ; \mathrm{RNLmax}[\mathrm{L}, \mathrm{j}]:=\operatorname{rimax} ; \mathrm{RNLmin}[\mathrm{L}, \mathrm{j}]:=\operatorname{rimin} ;$

$\operatorname{Cnorm}(\mathrm{L}, \mathrm{j})$;

if $\mathrm{E}>$ Emin then inc $(\mathrm{j})$;

end;

$\mathrm{s} 0:=\mathrm{s} 1$

until E<Emin;

$\operatorname{EnMax}[\mathrm{L}]:=\mathrm{j}-1$;

mods: $=\operatorname{mods}+\operatorname{EnMax}[\mathrm{L}]$

if $\operatorname{EnMax}[\mathrm{L}]=0$ then begin $\mathrm{Lmax}:=\mathrm{L}$; break; end;

end;

end;

$/ /++++++++++++++++++++++++++++++++++++$

$/ /+++$ Interface

procedure TForm1.PlotClear;

var i: integer;

begin

With Canvas do begin

Canvas.Brush.Color := clWhite;

Canvas.FillRect(Rect $(10,213,10+380,213+350))$;

Canvas.FillRect(Rect $(400,213,400+380,213+350)$ ); 
end;

end;

procedure TForm1.FormPaint(Sender: TObject);

var i: integer;

begin

PlotClear;

for $\mathrm{i}:=1$ to 25 do begin

StringGrid1.Cells $[\mathrm{i}, 0]:=' \mathrm{l}=$ '+ $\operatorname{IntToStr}(\mathrm{i}-1)$;

StringGrid1.Cells $[0, \mathrm{i}]:=' \mathrm{n}='+\operatorname{Int} \operatorname{ToStr}(\mathrm{i}-1)$;

end;

end;

procedure TForm1.PlotPsi(L,n:integer); // plot field

var $i, j$, npix:integer;

begin

$\operatorname{Init}(\mathrm{L}, \mathrm{ENL}[\mathrm{L}, \mathrm{n}])$;

npix:=Round $\left(1.1^{*} \mathrm{R} 4^{*} 1 \mathrm{E} 6 / 330\right)$;

PlotClear;

With Canvas do begin

Pen.Color:=clBlue;

Pen.Style:=psSolid;

Pen.Width: $=1$;

if $\mathrm{L}>0$ then MoveTo(20,543);

if $\mathrm{L}=0$ then begin $\mathrm{DU}(\mathrm{L}, \mathrm{ENL}[\mathrm{L}, \mathrm{n}])$; MoveTo(20,543-Round((yi*yi/YNL[L,n] $\left.\left.\left.{ }^{*} 310\right)\right)\right)$;

end;

for $\mathrm{j}:=$ Round(RNLmin[L,n]*1E6/npix) to 330 do 
begin

repeat DU(L, ENL[L,n]); until ri*1E6/npix >j;

LineTo(20+j,543-Round((yi*yi/YNL[L,n]*310)));

if $\mathrm{ri}>\mathrm{RNLmax}[\mathrm{L}, \mathrm{n}]$ then break;

end;

LineTo(20+Round(1.08*R4*1E6/npix),543);

Pen.Color:=clMedGray;

MoveTo(20+Round(R1*1E6/npix),213);

LineTo(20+Round(R1*1E6/npix),563);

MoveTo(20+Round(R2*1E6/npix),213);

LineTo(20+Round(R2*1E6/npix),563);

MoveTo(20+Round(R3*1E6/npix),213);

LineTo(20+Round(R3*1E6/npix),563);

MoveTo(20+Round(R4*1E6/npix),213);

LineTo(20+Round(R4*1E6/npix),563);

end;

end;

procedure TForm1.PlotPsi2(L,n:integer);

var i,j,cl,xp,yp,PiL,npix:integer;

tet,yr: extended;

begin

$\operatorname{Init}(\mathrm{L}, \mathrm{ENL}[\mathrm{L}, \mathrm{n}])$;

npix:=Round $(\mathrm{R} 4 * 1 \mathrm{E} 6 / 170)$;

With Canvas do begin

for $i:=\operatorname{Round}\left(\mathrm{RNLmin}[\mathrm{L}, \mathrm{n}]^{*} 1 \mathrm{E} 6 / \mathrm{npix}\right)$ to $170 \mathrm{do}$

begin 


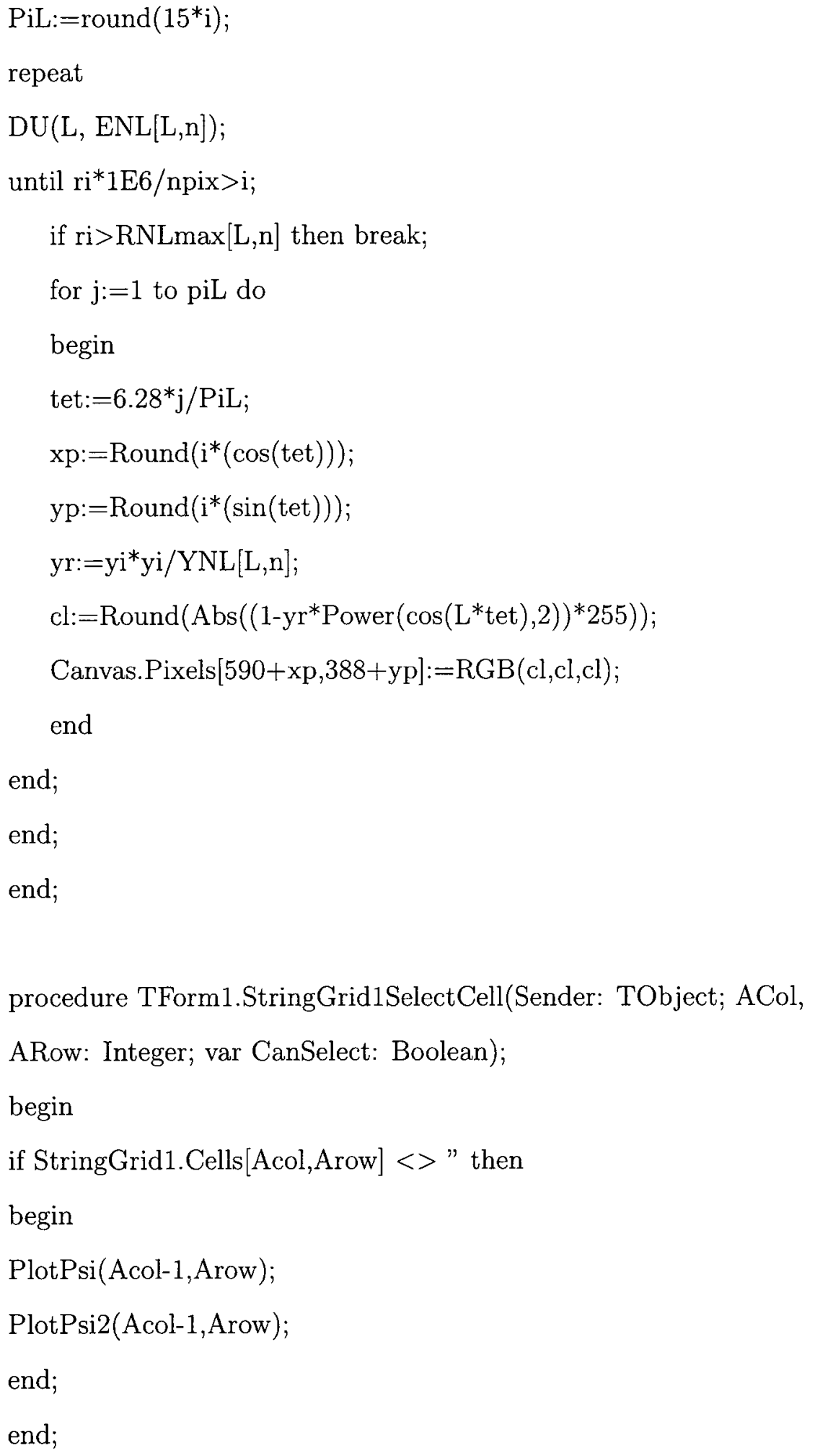




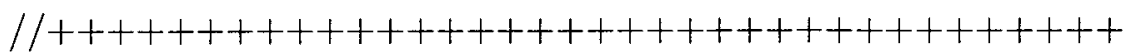

$1 /+++$ Save Data

procedure TForm1.SaveN(l:integer;E:real);

var

ri: real;

s: string;

fl:TFileStream;

begin

$\mathrm{s}:="$

ri: $=0.001$;

$\mathrm{fl}:=$ TFileStream.Create('C:/plasmon.txt',fmCreate);

fl.Position: $=0$;

while $\mathrm{ri}<\mathrm{R} 4^{*} 1.1$ do

begin

$\mathrm{ri}:=\mathrm{ri}+0.1$;

$\mathrm{s}:=\mathrm{s}+$ Float $\operatorname{ToStr}(\mathrm{ri})+{ }^{\prime},+$ FloatToStr$(\operatorname{Round} \operatorname{To}(\mathrm{kr}(\mathrm{ri}, \mathrm{E}, \mathrm{l}),-4))+\# 13 \# 10 ;$

end;

fl.Write(pointer(s), length(s));

fl.Destroy;

end;

procedure TForm1.SavePsi(r,y:real);

var

s: string;

fl:TFileStream; 
begin

if FileExists('C:/psi.txt')

then $\mathrm{fl}:=$ TFileStream. Create('C:/psi.txt',fmOpenWrite+fmShareDenyNone)

else $f:=$ TFileStream.Create('C:/psi.txt',fmCreate);

fl.Position:=fl.Size;

$\mathrm{s}:=\mathrm{s}+$ FloatToStr$(\operatorname{Round} \mathrm{To}(\mathrm{ri},-3))+{ }^{\prime}, '+$ FloatToStr$(\mathrm{yi})+\# 13 \# 10$;

fl.Write(pointer(s), length(s));

fl.Destroy;

end;

$/ /++++++++++++++++++++++++++++++++++++$

$/ /+++$ Test

procedure TForm1.testClick(Sender: TObject);

$\operatorname{var} \mathrm{i}, \mathrm{j}, \mathrm{L}:$ integer;

begin

Start;

Efind;

for $\mathrm{i}:=1$ to 25 do for $\mathrm{j}:=0$ to 25 do StringGrid1.Cells[j,i]:= ";

for $L:=0$ to $\operatorname{Lmax}$ do for $i:=1$ to EnMax $[L]$ do

StringGrid1.Cells $[\mathrm{L}+1, \mathrm{i}]:=$ floattostr(ENL $[\mathrm{L}, \mathrm{i}])$;

for $\mathrm{i}:=1$ to 25 do begin StringGrid1.Cells $[\mathrm{i}, 0]:=' \mathrm{l}={ }^{\prime}+\operatorname{IntToStr}(\mathrm{i}-1)$;

StringGrid1.Cells[0,i]:='n='+IntToStr(i-1);

end;

Label10.Caption:= InttoStr(mods)+' mods';

end;

end. 